\title{
Review of CMOS image sensors
}

\author{
M. Bigas ${ }^{\mathrm{a}}$, E. Cabruja ${ }^{\mathrm{a}, *}$, J. Forest $^{\mathrm{b}}$, J. Salvi ${ }^{\mathrm{b}}$ \\ ${ }^{\mathrm{a}}$ Centre Nacional de Microelectrònica, IMB-CNM (CSIC), Campus Universitat Autónoma de Barcelona, 08193 Bellaterra, Barcelona, Spain \\ ${ }^{\mathrm{b}}$ Institut d'Informàtica i Aplicacions Campus Montilivi, Universitat de Girona, 17071 Girona, Spain
}

Received 28 October 2004; received in revised form 29 June 2005; accepted 5 July 2005

Available online 6 September 2005

\begin{abstract}
The role of CMOS Image Sensors since their birth around the 1960s, has been changing a lot. Unlike the past, current CMOS Image Sensors are becoming competitive with regard to Charged Couple Device (CCD) technology. They offer many advantages with respect to CCD, such as lower power consumption, lower voltage operation, on-chip functionality and lower cost. Nevertheless, they are still too noisy and less sensitive than CCDs.

Noise and sensitivity are the key-factors to compete with industrial and scientific CCDs. It must be pointed out also that there are several kinds of CMOS Image sensors, each of them to satisfy the huge demand in different areas, such as Digital photography, industrial vision, medical and space applications, electrostatic sensing, automotive, instrumentation and 3D vision systems.

In the wake of that, a lot of research has been carried out, focusing on problems to be solved such as sensitivity, noise, power consumption, voltage operation, speed imaging and dynamic range. In this paper, CMOS Image Sensors are reviewed, providing information on the latest advances achieved, their applications, the new challenges and their limitations. In conclusion, the State-of-the-art of CMOS Image Sensors. (C) 2005 Elsevier Ltd. All rights reserved.
\end{abstract}

Keywords: CMOS image sensors; APS

\section{Introduction}

\subsection{Introduction}

Currently, there are many different Imaging Systems suitable for different purposes, de pending upon their final application. Digital Cameras, Camcorders, Webcams, Security cameras or IR-cameras are well-known Imaging Systems. Moreover, as the purposes are different, the technologies used differ from each other. This situation has been possible thanks to the fact that Imaging Technologies, mainly the ones concerning CMOS imagers, have been improving their performance, their functional capability and their flexibility during last years.

CMOS image sensors have received much attention over the last decade, because their performance is very promising compared to CCDs. New horizons can be opened, like ultra

\footnotetext{
* Corresponding author. Tel.: + 34 935947700; fax: + 34935801496.

E-mail addresses: marc.bigas@cnm.es (M. Bigas),enric.cabruja@cnm. es (E. Cabruja).
}

0026-2692/\$ - see front matter (C) 2005 Elsevier Ltd. All rights reserved. doi:10.1016/j.mejo.2005.07.002 low power or camera-on-chip systems. Owing to this situation and the latest developments within this field, this paper reviews CMOS image sensors since 1997 in order to continue and update the review reported by E. Fossum [1].

In order to understand why CMOS image sensors have emerged as a strong alternative to CCDs, it is important, first, to highlight the Advantages and Disadvantages of CMOS image sensors.

\subsection{Advantages and disadvantages}

The main Advantages of CMOS imagers are:

1. Low power consumption. Estimates of CMOS power consumption range from one-third to more than 100 times less than that of CCDs [2]. Besides, they work at low voltage. CMOS imagers only need one supply voltage, instead of CCDs, which need 3 or 4 .

2. Lower cost compared to CCD's technology.

3. On chip functionality and compatibility with standard CMOS technology. CMOS imagers allow monolithical integration of readout and signal processing electronics. In 2001, a study for Cross Contamination between CMOS Image Sensor and IC product showed no 
problems [3]. A sensor can integrate various signal and image processing blocks such as amplifiers, ADCs, circuits for colour processing and data compression, etc. on the same chip.

4. Miniaturisation, although important limitations exist, the level of integration is rather high [4].

5. Random access of image data.

6. Selective read-out mechanism $[4,5]$

7. High-speed imaging. The flexibility and the possibility to acquire images in a very short period of time [6].

8. To avoid blooming and smearing effects, which are typical problems of CDD technology [6].

As outlined before, despite these advantages, there are still significant Disadvantages of CMOS image sensors compared to CCD technology. Therefore, these problems need to be solved so that CMOS image sensors can compete in any area. These disadvantages are:

1. Sensitivity: The basic quality criterion for pixel sensitivity is the product of its Fill Factor and its Quantum Efficiency (FFxQE) where Fill Factor is the ratio of light-sensitive area to the pixel's total size, and Quantum efficiency is the ratio of photon-generated electrons that the pixel captures to the photons incident on the pixel area. It must be pointed out that Active Pixel Sensors (APS) have reduced sensitivity to incident light, due to a limited Fill Factor, hence, less quantum efficiency.

2. Noise: CMOS Image sensors suffer from different noise sources which set the fundamental limits of their performance, especially under low illumination.

3. Dynamic range (DR): Dynamic Range, which is the ratio of the saturation signal to the rms noise floor of the sensor, is limited by the photosensitive-area size, integration time and noise floor.

4. Less image quality than CCD.

In order to overcome the disadvantages outlined before and, also, to improve the current advantages as well, the research on CMOS image sensors, since 1997, has been mostly focused on the following areas:

- Low noise

- High dynamic range

- High sensitivity and High fill factor

- Low power consumption

- Low voltage operation

- High speed imaging

In spite of the high number of applications of the imaging systems, all of them have almost always the same basic functions: (1) Optical gathering of photons (lens), (2) Wavelength discrimination of photons (filter), (3) Detector for photons to electrons conversion (photodiode), (4) A method to readout the detector (CCD), (5) Timing, control, and drive electronics for the sensors, (6) Signal processing electronics such as for Correlated Double Sampling (CDS) or for color processing, (7) Analog-todigital conversion, (8) Interface electronics.

\subsection{Historical background}

\subsubsection{Before 1997}

CMOS image sensors could not compete in the past with CCD technology, although the first solid-state imagers presented in the $60 \mathrm{~s}$ and early $70 \mathrm{~s}$ used MOS diodes as light sensitive elements and during the $60 \mathrm{~s}$ several works were performed in the solid-state image sensor's field, using NMOS, PMOS and bipolar processes. [1] For instance, photodiode image sensors with MOS scanning circuits were known from mid $60 \mathrm{~s}$. However, they were not embraced commercially because of poor performance and large pixel size (for that time) relative to that of the CCDs. In fact, even though CMOS image sensors appeared in 1967, CCDs have prevailed since their invention in 1970 [7]. Full-analog CCDs have dominated the vision applications owing to their superior dynamic range, lower fixed-pattern noise (FPN), smaller pixels and higher sensitivity to light [2].

In the early 1990s, CMOS image sensors re-emerged as an alternative to CCDs thanks to the advantages pointed out before. Passive pixel CMOS arrays were the first generation. Major improvements in signal-to-noise ratio for photodiodes and charge-injection devices (CIDs) could be made by adding an amplifier per column or per row. Therefore, sensors that implement a buffer, which acts as simple source follower, per pixel have been known as active-pixel sensors (APS) and represent the second generation of CMOS imagers [8]. CMOS APS (Active Pixel Sensors) promised to provide: lower noise readout, improved scalability to large array formats and higher speed readout compared to PPS.

\subsubsection{After 1997}

Recently, the research has been focusing, mainly, on the improvement of the APS, because APS are the pixel circuit that have shown better performance and flexibility.

In order to strongly compete with CCD technology, the aim of researchers has been to obtain higher performance imaging systems based on CMOS technology. Therefore, there have been several reports on improving the fill-factor (FF) with low power consumption, low voltage operation, low noise, high speed imaging and high dynamic range. Moreover, little research has been done on other topics such as pixel shape optimization [9], pixels on SOI substrate [10], high resolution [11], APS with variable resolution [12,13], self-correcting [14,15] and for low light [16-18], etc.

On the other hand, new applications have emerged due to the CMOS imager development. Automotive applications, imaging for cellular or static phones, computer video, space, medical, digital photography and 3D applications have been improved. So many applications areas caused that CMOS 
technology made a breakthrough on two fronts in 2000: sensors for computers and cell phones on the low end, and ultra high speed, large format imaging on the high-end [19]. Moreover, new technologies and architectures appeared due to scale effects. For instance, Thin Film on ASIC (TFA) technology [20-24] and Complementary Active Pixel Sensors (CAPS) [25-31]. Finally, some studies have been carried out to study the radiation effects and how radiation induced dark current in APS [32-39] and the effect of hot carriers [40]. In addition, it is well known that heavy metals such as $\mathrm{Cu}, \mathrm{Ni}, \mathrm{Fe}$ or $\mathrm{Zn}$, which appear in some CMOS image sensor processes, can cause defects in silicon and influence gate oxide quality in VLSI circuits. So the crosscontamination between CMOS image sensor and IC technologies has been studied as well [3].

\subsection{CCD technology limitations}

CCD technology has prevailed since its invention in 1970, because it provided better solutions to the typical problems, such as FPN and it had a higher fill factor, smaller pixel size, larger format, etc. than CMOS, which could not compete with CCD performance. Then, the research has been mainly focused on CCD technology. Nevertheless, CCD technology has some limitations: For instance, in a CCD-based system, the basic function often consumes several watts (1-5 W) and is, therefore, a major drain for the battery. Furthermore, unlike CMOS image sensors, CCD cannot be monolithically integrated with analog readout and digital control electronics [1]. New applications have appeared as well. For instance, in the automotive field, the image sensor has to fulfill the specifications concerning the temperature range, the range of illumination, and the power dissipation. CCD image sensors cannot guarantee their functionality over the whole temperature range required, or to cover all lighting conditions during daytime. They cannot operate beyond quite restrictive ranges of illumination and temperature. For instance, a non illuminated $\mathrm{CCD}$ is completely full of electrons after roughly $1 \mathrm{~min}$ at room temperature and the dark current doubles approximately every $7 \mathrm{~K}$. This means that noise drastically increases with the temperature of the chip.

Also there is a need to acquire images in a very short time for high speed applications, therefore short integration time is required. This leads to image sensors equipped with synchronous shutters in order to avoid blur [6]. Other CCD typical problems are: Blooming and smearing [6]. CCDs are high capacitance devices, so they suffer from high power dissipation. CCDs need many different voltage levels, they are sensitive to radiation and their readout rate is limited.

\subsection{CMOS Image sensors (APS) as an alternative to overcome CCDs limitations}

CMOS imagers began to be a strong alternative since early $90 \mathrm{~s}$ (see Fig. 1). Their most important feature was that they would satisfy the demand for low-power, miniaturised and cost-effective imaging systems. Moreover, CMOS image sensors offered the possibility to monolithically integrate a significant amount of VLSI electronics on-chip and reduce component and packaging costs [1].

However, passive pixel CMOS arrays were the first generation. CMOS Active Pixel Sensors (APS) have offered better performance though. Up to now, a lot of research and studies has been done on this topic and CMOS APS technology has demonstrated noise, quantum efficiency, and dynamic range performance comparable to CCDs [41]. However, CCDs still offer a better image quality, especially for digital still applications. Therefore, CCDs are still superior to CMOS image sensors as far as signal-to-noise and dynamic range is concerned. This means that CCDs are still the first choice for high quality still photography.

In spite of the huge work that has been carried out in this area, more research on reducing the noise and increasing the sensitivity of the CMOS imagers is needed in order to compete with industrial and scientific CCDs. For instance, if the noise issues (mainly reset noise and dark current shot noise) can be solved with CMOS imagers, they might be able to challenge CCDs in digital still applications [5].

Thanks to the fact that there were niches to cover such as high-speed, motion analysis or detection, etc. [42], CMOS APS technology has been growing up and, currently, there are different kinds of pixel circuits depending on their purpose. For instance, logarithmic APS, capacitive transimpedance amplifier (CTIA) APS [43], APS with shutter [44] or complementary active pixel sensors CAPS [31]. Currently, there is no CMOS image sensor that can provide the global quality of a CCD in terms of noise, sensitivity, dynamic range and so on. This means that it is possible to

\begin{tabular}{|c|c|}
\hline CCD & CMOS \\
\hline Lower noise & Low power consumption \\
\hline Smaller pixel size & Single power supply \\
\hline Lower dark current & High Integration capability \\
\hline $100 \%$ Fill Factor & Lower cost \\
\hline Higher sen sitivity & Single master clock \\
\hline Electronic shutter without artifacts & Random access \\
\hline
\end{tabular}

Fig. 1. Summary of the main advantages of CCD and CMOS image sensors 
reach or improve one or two of their characteristics with a specific architecture, but not all together.

\subsection{New applications-new challenges}

The improvement of CMOS image sensors has opened up new application areas [45], owing to the lower cost of CMOS image sensors. They can compete with CCDs in applications such as IR-vision (systems for automobile drivers under fog and night driving conditions, security cameras, baby monitors that can 'see' in the dark, etc.)

Besides these, imaging or vision systems for X-ray, space, medical, 3D, consumer electronics, automotive or low-light applications are required, and most of them need highly integrated imaging systems, so CMOS image sensors are well situated to jump into the market.

Moreover, imaging or vision systems have to offer, in order to be ideal, good imaging performance with low noise, no lag, no smear, good blooming control, random access, simple clocks and fast readout rates.

\subsection{CMOS Image sensors limitations and device scaling considerations}

\subsubsection{Industry trend}

The technology has advanced from a $2 \mu \mathrm{m}$ CMOS in 1993 to $0.25 \mu \mathrm{m}$ in 1996 [4] and less than $0.1 \mu \mathrm{m}$ will also be possible. So, considering scaling effects has been necessary in order to know where and which are the limits of CMOS imagers.

Some Scaling Considerations were studied in 1996[46] and in 1997[4]. The question was whether the image sensing performance of CMOS imagers would get better or worse as the technology would be scaled. The question arose because if CMOS imagers would scale down as fast as industry standard CMOS technologies, CMOS imagers would achieve a smaller pixel size than CCDs during the following years [4]. Anyway, it seemed to be clear that 'standard' CMOS technology, which provided good imaging performance at $2-1 \mu \mathrm{m}$ without any process change, would need some modifications in its fabrication process and innovations on the pixel architecture in order to enable CMOS imagers to perform good quality imaging when using the $0.25 \mu \mathrm{m}$ generation technology and beyond [1]. In fact, CMOS imagers could not be scaled down using standard CMOS technology because scaling effects increase leakage current and reduce dynamic range. That is to say that performance was getting worse. Thus, technological changes in CMOS technology are needed in order to reach the imaging performance of CCDs with a CMOS imager.

In 2000 [20] a scaling perspectives study was done and, certainly, new technological processes appeared, such as PPD [20] and TFA imagers [20-24] (See Fig. 2). Later, CAPS appeared as well [25-31]. TFA imagers are immune to negative scaling impacts on sensitivity. Even more, they

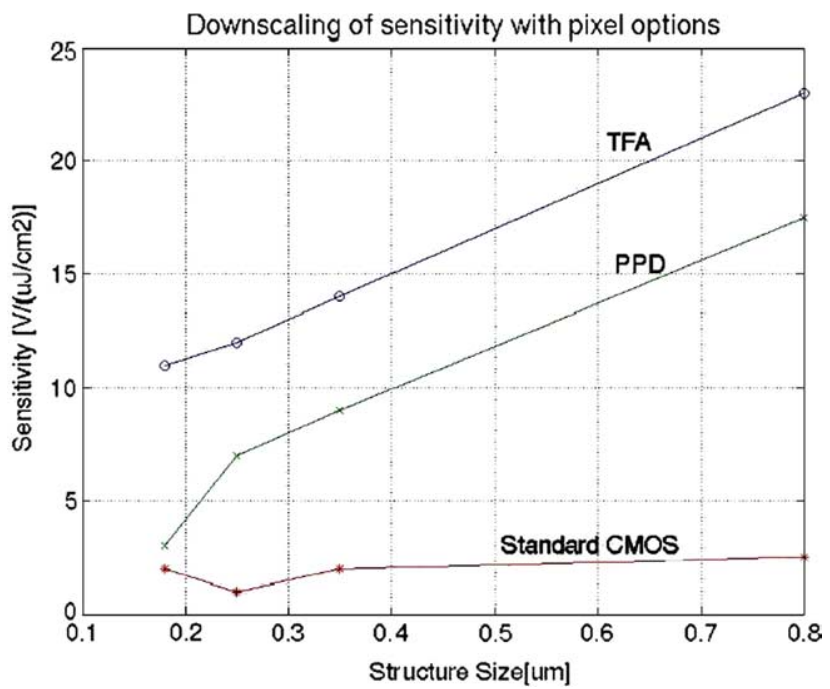

Fig. 2. Downscaling of sensitivity with pixel options.

offer high sensitivity and high dynamic range. Nevertheless this technology is not suitable down to $0.1 \mu \mathrm{m}$.

Thus, it has been demonstrated the limitation of conventional APS, because conventional pixel architecture (APS) cannot work properly with a $0.1 \mu \mathrm{m}$ technology or below because the low power of these technologies implies a decrease in the saturation level and in the light sensitivity that it is not acceptable. Nevertheless, an alternative architecture called CAPS (Complementary APS) came on scene [25-31]. They are a possible way to design a highly integrated, high performance CMOS image sensor in the deep sub-quarter micron technology, because CAPS architecture has a very attractive low-voltage operation capability. For instance $1.0 \mathrm{~V}[25,26,29,30]$ Moreover, the possibility to reach low power and low voltage consumption depends on the capability to scale down the current technology.

\section{CMOS image sensors}

CMOS image sensors are mixed-signal circuits containing pixels, analog signal processors, analog-to-digital converters, bias generators, timing generators, digital logic and memory.

\subsection{Overall architecture}

There are several CMOS imager topologies depending on their purpose. Nevertheless, CMOS imagers architecture can be divided into four main blocks, as Fig. 3 shows.

1. Pixel Array

2. Analog Signal Processors

3. Row and Column Selector

4. Timing and Control 


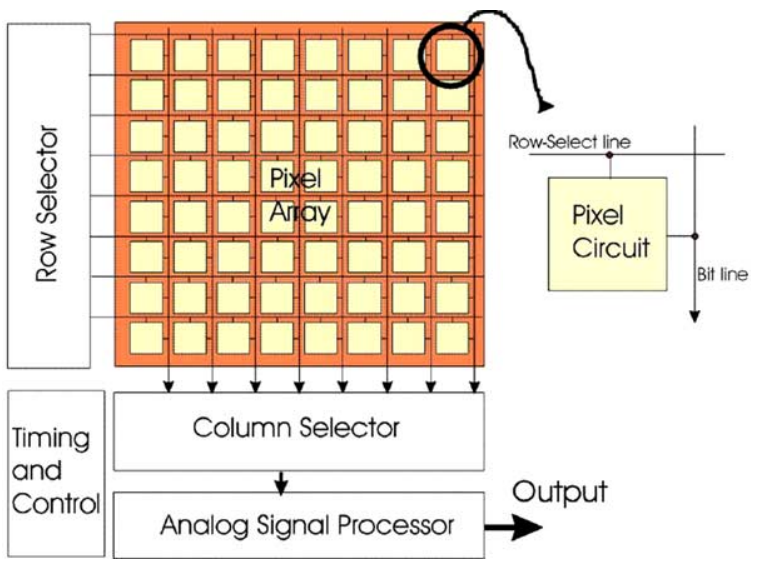

Fig. 3. CMOS image sensor floorplan.

\subsection{Pixel circuits}

\subsubsection{Traditional imagers or photodetectors}

1. Photodiodes: Semiconductor devices responsive to high energy particles and photons. They operate by absorption of photons or charged particles and the collected electrons which decrease the voltage across the photodiode in a proportional basis to the incident power. Currently, photodiode CMOS pixels are the most popular ones.

2. Photogates (PG) or CID (Charge-injection device): Semiconductor devices that also collect the photongenerated electrons, but only when the photogate is biased to a high potential.

3. CCD (Charge-coupled device): Device architecture based on series and parallel connection of capacitors, which are made using a dedicated semiconductor process.

\subsubsection{Pixel circuits}

Pixel circuits are mainly divided into active pixels (APS) and passive pixels (PPS). APS are sensors that implement a buffer per pixel. This buffer is as simple as adding a sourcefollower. Currently, APS are the predominant devices, although in some cases PPS are also used.

\subsubsection{Passive pixels (PPS). They were the first CMOS} imagers. They are based on photodiodes without internal amplification. In these devices each pixel consists of a photodetector (e.g. photodiode), and a transistor in order to connect it to a readout structure (See Fig. 4). Then, after adressing the pixel by opening the row-select transistor (RS), the pixel is reset along the bit line and RS. In spite of the small pixel size capability and a large fill factor, they suffer from low sensitivity and high noise [20] due to the large column's capacitance with respect to the pixel's one.

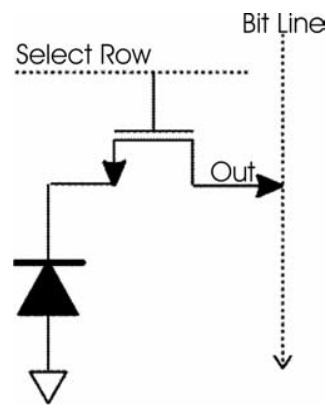

Fig. 4. A photodiode-type PPS schematic.

2.2.2.2. Active pixels (APS). APS are sensors that implement a buffer per pixel. This buffer is a simple source-follower. It is well known that the insertion of a buffer/amplifier into the pixel improves the performance of the pixel. Power dissipation is minimal and, generally, less than CCD's, because each amplifier is only activated during readout. Nevertheless, it must be noted APS technology has some disadvantages: Conventional APS suffer from a high level of fixed pattern noise $(F P N)$ due to wafer process variations that cause differences in the transistor thresholds and gain characteristics. A solution is to use a Correlated Double Sampling $(C D S)$ circuit, which can almost eliminate the threshold variations that cause offsets in the video background.

2.2.2.3. Photodiode (PD) type APS. The photodiode-type (PD) APS is considered as standard and it was described by Noble in 1968 [1,20] (See Fig. 5a). It consists of threetransistor: a reset transistor, for resetting the photodiode voltage; and a source follower with select transistor, for buffering the photodiode voltage onto a vertical-column bus. The PD APS is suitable for most mid low-performance applications.

2.2.2.4. Photogate( $P G)$ type APS. It was introduced later than PD APS, in $1993[1,20]$ and it employs the principle of operation of CCDs concerning integration transport and readout inside each pixel. Its transfer of charge and correlated double sampling permits a low noise operation. Thus, it is suitable for high performance and low light applications. A typical schematic is shown in Fig. 5c.

2.2.2.5. Logarithmic APS. Non-linear output of the sensor can be desirable. This fact permits an increase on the intrascene dynamic range. Logarithmic APS are suitable for High Dynamic Range applications, although they suffer from large FPN. Owing to this fact, currently, they are not as used as before. It must be pointed out that they are used a lot in silicon retinas. A typical schematic is shown in the Fig. 5b.

2.2.2.6. CTIA APS pixels. As highlighted before, conventional APS suffer a high level of FPN. Thus, reducing FPN has been a challenge for quite a while and some solutions 
(a)

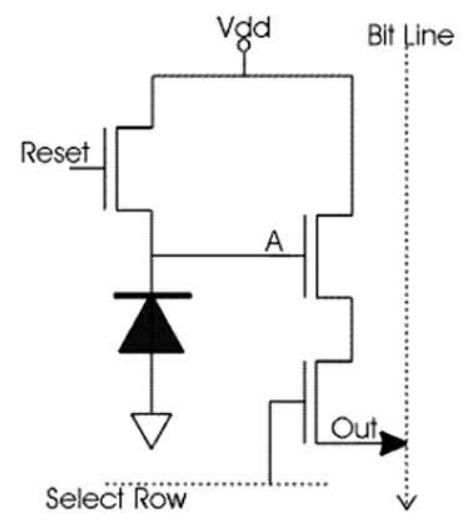

(c)

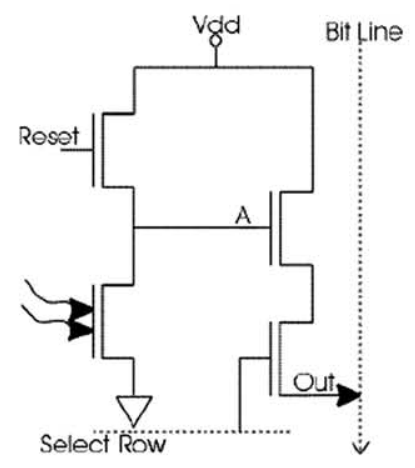

(e)

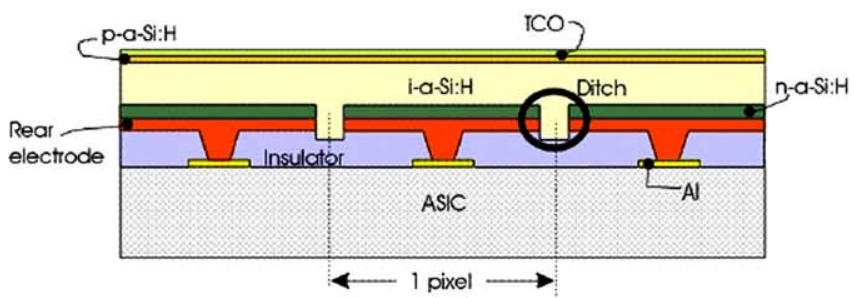

(g)

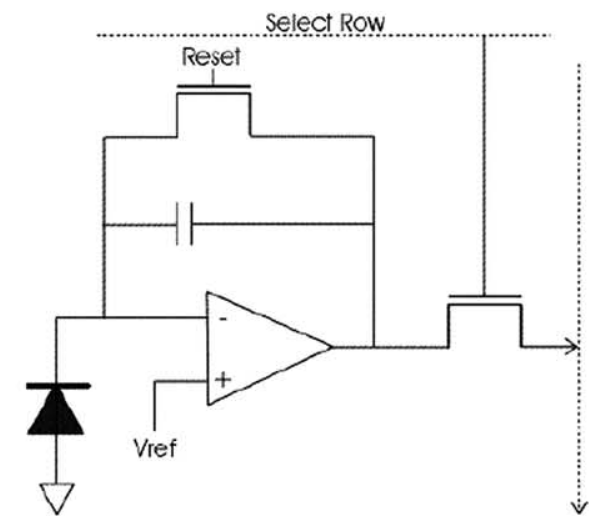

(b)
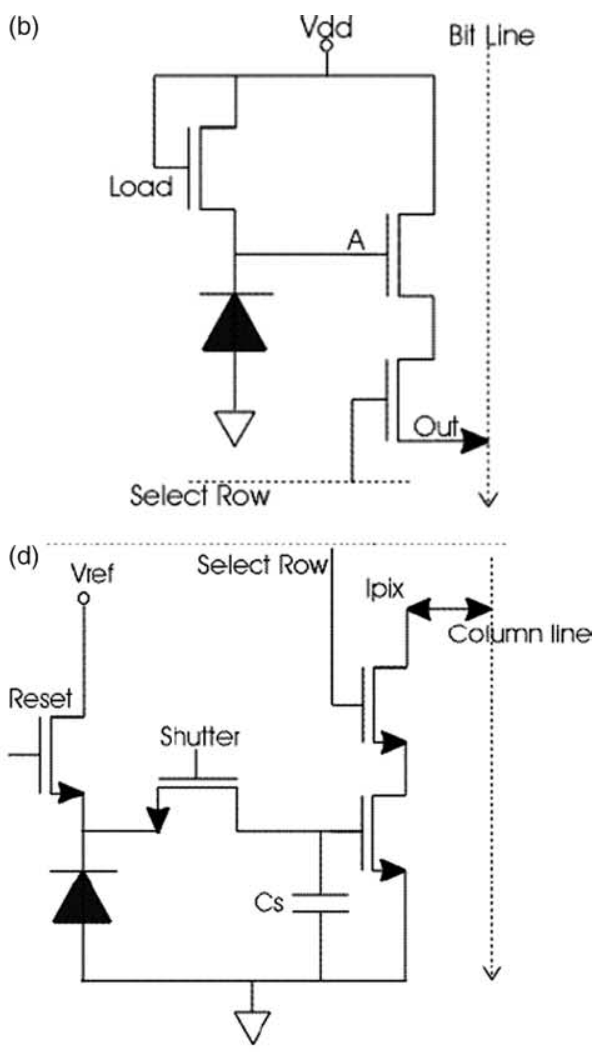

(f)
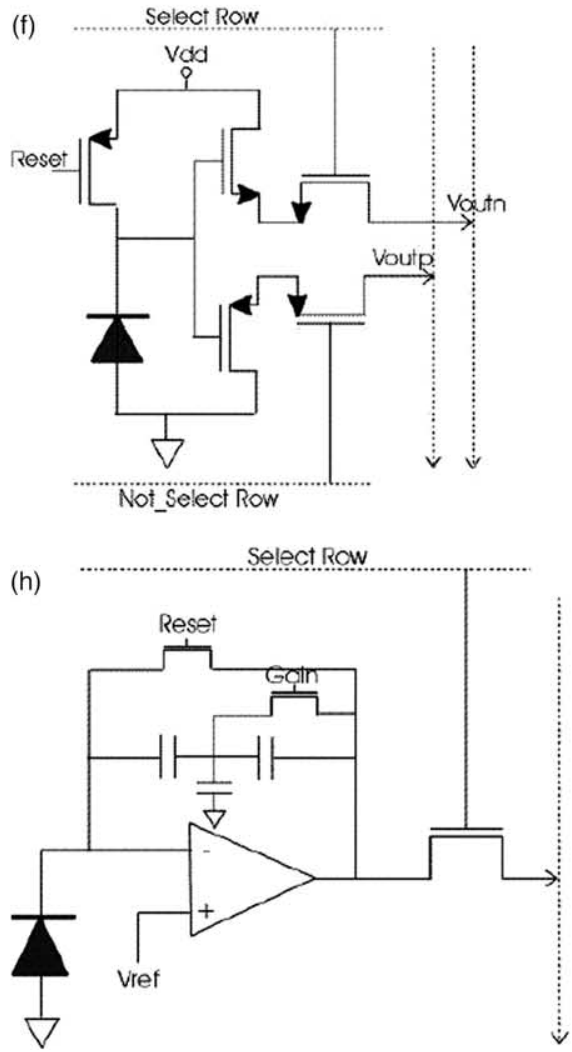

Fig. 5. (a) A photodiode- type APS schematic, (b) A photodiode- type logarithmic APS schematic, (c) A photogate- type APS schematic, (d) Photodiode- type shutter APS schematic, (e) TFA pixel, (f) A photodiode- type CAPS schematic, (g) A photodiode- type Low FPN CTIA APS schematic (h) A photodiode- type High FPN CTIA APS schematic. 
have been reported. For instance, capacitive transimpedance amplifier (CTIA) pixels can achieve low FPN [43]. Besides this, the high gain and low read noise are advantages of using CTIAs as well. Fig. 5h and g show a high FPN and a low FPN CTIA APS pixel schematics respectively.

\subsubsection{Pinned photodiode (PPD) pixel. The pinned} photodiode [20], which was previously used in chargecoupled devices, was proposed early during the development of CMOS active-pixel image sensors. Besides lower pixel noise, the pinned photodiode offers reduced dark current. Therefore, they are a PG's alternative, because their architecture offers higher sensitivities than PG.

2.2.2.8. TFA pixels. The thin film on ASIC (TFA) pixels were developed in order to improve sensitivity [20-24]. They consist of an amorphous silicon (a-Si:H) multilayer system that is deposited on top of an ASIC.

Their absorption coefficient for visible light is approximately 20 times bigger than crystalline's silicon (c-Si). In fact, TFA pixels are suitable for High Dynamic Range applications. A typical TFA structure pixel is shown in Fig. 5e.

\subsubsection{Complementary active pixels sensors CAPS. CAPS} are a possible way to manufacture a highly integrated, high performance CMOS image sensor in the deep sub-quarter micron technology. Furthermore, CAPS architecture has low power consumption and a high low-voltage operation capability. A typical schematic is shown in Fig. 5f. The main new features are that the reset transistor is replaced by a PMOS. In addition, a complementary signal path is implemented and the pixel gives out two signal path outputs: $V_{\text {outn }}$ and $V_{\text {outp }}$ [25-31].

2.2.2.10. Other pixels. There is more pixel architectures [47], due to the huge number of possible applications. For instance, pixel circuits suitable for high speed operation by adding a shutter transistor [44] (see Fig. 5d). Also Diericks (Fillfactory) introduced a novel 100\% fill factor pixel.

Overall, PG, PPD and TFA are detector structures to improve the sensitivity. Nevertheless, TFA provides significantly better values than PPD due to the higher fill factor and higher quantum efficiency [20].

\subsection{Analog signal processing}

Analog signal processing circuits are used in order to improve the performance and functionality of CMOS image sensors. However, they tend to involve less pixel density and increase the chip area due to the added functions. Some research has been done to overcome these problems [48].

Firstly, there are some well known traditional signal processing systems. For instance, additional analog-signalprocessing circuitry located at the periphery of the array permits the suppression of both, temporal and fixed-pattern noise. As an example, Correlated Double Sampling (CDS) or Double Differencing Sampling (DDS) suppress FPN [49, 43,50,51]. Others achieve high SNR [52] and ADC for camera-on-a-chip. Secondly, there are also several signal processing systems depending on the application. For instance, signal processing such as K-winners-take-all, are suitable for 3D vision systems [53-58] and subpixel accuracy [59-65]. Smoothing [66], motion detection [6769], programmable amplification, multiresolution imaging [70], video compression [71], dynamic range enhancement, discrete cosine transform (DCT), intensity sorting, etc. are other signal processing systems.

\subsection{Readout methods}

Readout methods have an important influence in the sensor performance. Thus, there are several readout methods depending on the desirable application. The main requirements are:

1. low power dissipation

2. high resolution

3. good linearity

4. stable detector bias

5. low noise

6. high injection efficiency

7. small pixel size

8. good dynamic range

APS readout structures fulfil 4 and 8 requirements, but not 3 and 7. On the other hand PPS readout structure fulfils 7 and not 3, 4, 6 and 8. Finally, share-buffered direct-injection (SBDI) [72] readout structure combine both imagers characteristics.

Therefore, it is possible to find suitable traditional readout methods for low FPN [73-76], for high frame rates [73], to increase linearity and DR [72], with high SNR and ultrahigh- sensitivity [77,78] and for infrared detectors [79]. Finally, there are also specialised readout methods suitable for ultra high sensitivity, for focal plane array, for $\mathrm{X}$-ray imaging, for an emission-transmission medical imaging systems, for low-light levels, detectors with selftriggered readout, offset-free column readout circuit and transversal-readout architecture

\subsection{Noise sources}

CMOS Image sensors suffer from several noise sources. They set the fundamental limits on image sensor performance, especially under low illumination and in video applications. Therefore it is important to have an overview of all of them [80].

The noise sources in CMOS imagers can be divided into Temporal Noise [81] and Fixed Pattern Noise (FPN)[43]. 


\subsubsection{Temporal noise}

It can be divided into different kinds of noise, depending on its source:

- Pixel noise: photon shot noise, reset or kT/C noise (which is the thermal noise resulting from resetting after each pixel's readout. The $k$ is Boltzmann's constant, $T$ is the absolute temperature; and $C$ is the junction parasitic capacitance), dark current shot noise and MOS device noise (thermal, $1 / f$ or flicker, etc.)

- Column Amplifier noise

- Programmable gain amplifier noise

- ADC noise

- Overall temporal noise, noise floor or reading noise.

\subsubsection{Fixed pattern noise (FPN)}

It has been a huge CMOS imagers' limitation. FPN is the fixed variation in the output between pixels when a uniform input is applied. In a perfect image sensor, each pixel should have the same output provided that the same input is applied, but in current image sensors the output of each sensor is different. FPN does not change as a function of time and can be characterized, assuming a linear pixel response, as a variation in the offset and gain at each pixel.

$V i j(t)=G i j X i j(t)+O i, j$

V output,

$\mathrm{G}$ gain of pixel,

$\mathrm{X}$ input,

O offset of pixel,

Gain FPN pixel to pixel variation of Gij,

Offset FPN pixel to pixel variation of Oij.

\section{Latest developments in the field of CMOS imagers}

The research has been mainly focused on APS in areas like Low noise, High dynamic range, High sensitivity and High fill factor, Low power consumption, Low voltage operation, High speed imaging. To remark is that all these features are difficult to achieve in one design. Hence, depending on the application, one feature will have more priority than another one.

\subsection{High dynamic range (DR)}

The ratio of the saturation signal to the rms noise floor of the sensor is known as dynamic range. This is limited by the photosensitive-area size, integration time and noise floor, which is the noise generated in the pixel and the signal processing electronics. DR is limited by the integration time, although high dynamic range image readout can be achieved by using different exposure or integration times [82].
Secondly, with respect to the noise floor the use of a linear readout is more suitable than a logarithmic one. So Dynamic Range of CMOS APS is strongly managed by the readout method. Finally, the photosensitive-area size is an important issue because of the well-known scaling effects.

The major problem of artificial image acquisition has been the extraordinary high optical dynamic range of natural scenes. For instance, the human vision system exhibits an enormous optical dynamic range of about $200 \mathrm{~dB}$, due to the fact that it can adapt to an extremely high range of light intensity levels [83]. Nevertheless, artificial imagers have been much poorer in this aspect. With conventional CCD sensors it is hard to reach high dynamic range and CMOS imagers with logarithmic response suffer from excessive FPN and temperature drift. For instance, in year 2000 the conventional CCD imagers exhibited usually a DR of about 50-70 dB only and on the other hand, CMOS imagers would achieve better DR, up to $140 \mathrm{~dB}$, than CCDs [82], although they used logarithmic readout, which has some disadvantages such as a high FPN.

In fact, some research has been done to obtain CMOS image sensors with high dynamic range:

\subsubsection{Logarithmic}

The use CMOS imagers with logarithmic readout or the non-linear output of the logarithmic pixel provides higher dynamic range (see Fig. 6). Nevertheless, logarithmic response has a large FPN and slower response time for low light levels, which bring down the image quality. Thus, some systems based on logarithmic response have been developed in order to offer high DR with low FPN. For instance, in 1998, the University of Heidelberg proposed a CMOS camera chip with logarithmic response and selfcalibrating FPN correction [84]. Its results showed a significant FPN reduction. Fraunhofer Institute of Microelectronic Circuits and Systems of Duisburg suggested also a CMOS imager with Local Brightness adaptation [85]. It used logarithmic image sensors in order to reach high DR and FPN was also compensated. In year 2000, S. Kavadias reported a technique to remove the high FPN, due to its logarithmic response [86]. This CMOS image sensor, which

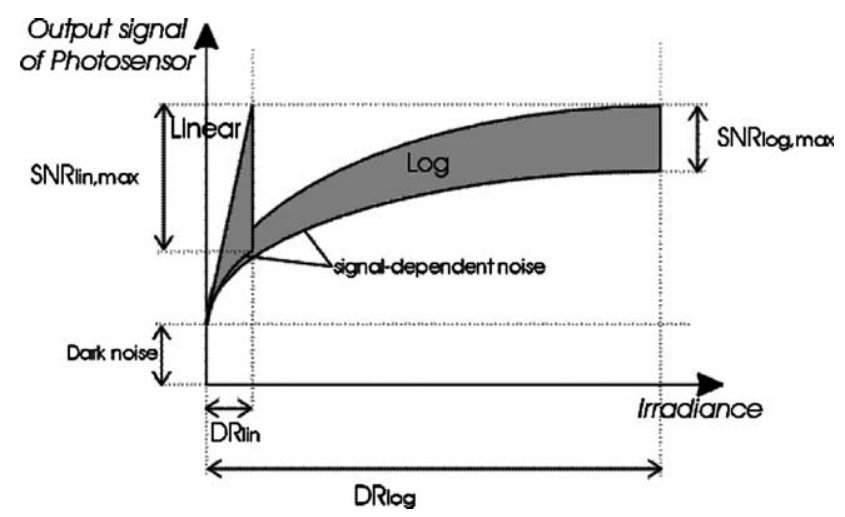

Fig. 6. Photoconversion characteristics: linear vs logarithmic. 
was based on an active pixel structure, employed on-chip calibration and achieved a DR of $120 \mathrm{~dB}$ and the FPN was $2,5 \%$ of the output signal range. Finally in year 2003 a multiresolution scheme and a cost-effective architecture for nonlinear analog-to-digital conversion was presented. These two features combined together improve the sensors quality under low light intensity [87].

\subsubsection{Linear}

On the other hand, using CMOS imagers with linear readout can improve FPN, even though they can achieve lower DR (see Fig. 6) than logarithmic ones. For instance, in 1999 a new design, a $1280 \times 1024$ digital CMOS image sensor with enhanced dynamic range, was reported [51]. The response was linear, low FPN was achieved and DR was of $69 \mathrm{~dB}$. In year 2000 a CMOS image sensor for automotive applications was proposed [83]. It offered a high dynamic range up to $120 \mathrm{~dB}$ and an excellent image quality due to its linear readout. Furthermore, it had good temperature behavior up to $85^{\circ} \mathrm{C}$. In year 2002 a high dynamic CMOS imager with spiking pixels, pixel-level ADC, and linear characteristics was reported [88]. It had a DR of $93 \mathrm{~dB}$, although $120 \mathrm{~dB}$ was expected. Finally, it is reported of a mechanism [82], using linear readout, capable for adjusting its sensitivity depending on the absolute illumination level, like human vision. This is reached by using different integration times. The results demonstrate that with 1 integration time, a DR of $61 \mathrm{~dB}$ is reached. In contrast, with two integration times, the DR is of $92 \mathrm{~dB}$.

\subsubsection{Combined}

In 1998, the University of Waterloo reported a CMOS APS with combined linear and logarithmic mode operation [89]. The results showed that it had good linearity, in the linear mode operation, and it reached wide dynamic range in the logarithmic mode.

So the issue is to determine which is the more convenient readout method in each application. Nevertheless, using linear readout methods with different integration times seems to be more suitable.

\subsubsection{TFA technology}

As outlined before, TFA technology [23,24] is suitable for achieving a high dynamic range and a high fill factor. In 1999-2000, T. Lulé reported a 100.000 pixel imager in TFA technology $[21,22]$. The main feature was that every pixel contained an automatic shutter, which adapted the integration time to the local intensity. This allowed obtaining a high DR of $120 \mathrm{~dB}$.

\subsection{High sensitivity (High fill-factor $(F F)$ and high quantum efficiency)}

\subsubsection{Background}

The basic quality criterion for pixel sensitivity is the product of its Fill Factor and its Quantum Efficiency
(FF $\times \mathrm{QE})$. Where Fill Factor is the ratio of light-sensitive area to the pixel's total size; also known as aperture efficiency, and Quantum efficiency is the ratio of photongenerated electrons that the pixel captures to the photons incident on the pixel area. Photons are lost for conversion due to: reflection on dielectrics, no absorption in the acquisition layer and loss of charges and recombination.

It is well known that a good image quality is obtained if most of the chip area is dedicated to the photodetectors. Therefore in order to achieve a good image quality, a high Fill Factor (FF) is needed. Unlike CCDs, which achieve around $100 \%$ FF [90,91], CMOS APS FF are limited, because each pixel has an area devoted to the CMOS readout circuitry. Around 30\% FF is a kind of standard. In CMOS APS pixel, the Fill Factor is limited by: (a) shadowing by metals or silicides, (b) collection of photons by the insensitive junctions of the active pixel, (c) the relatively small size of the useful photo-sensitive junction, (d) recombination of photo-generated carriers with majority carriers, limiting the diffusion length. Besides this, a high fill factor allows shorter exposure times for a given pixel size or smaller pixel sizes for a given sensitive area. Thus, FF plays an important role in the scaling perspectives and imager's performance.

In the wake of that, a lot of research has been done to increase the fill factor of CMOS APS. There are different methods used to improve the FF: one is to design active pixels with larger photodiodes, although small pixels can not be made and large photodiodes have low charge conversion sensitivity due to their higher capacitance [92]. The other method is to make passive pixels, but their performance with respect to active pixels is worse. On the other hand, microlenses, which help funneling photons to the light-sensitive portion of the pixel [93,1], are another alternative to overcome this problem. They could reach up to $90 \%$ Fill Factor. In spite of this $90 \%$ Fill Factor, they have some disadvantages such as the reduction of efficiency as microlens dimension decreases. In fact, some high fill-factor designs based on CMOS APS exist, which can enhance the FF: A. Bermak developed a $46 \%$ Fill Factor native logarithmic pixel [94] in $0.7 \mu \mathrm{m}$ CMOS technology in 2000. In 2002, the Georgia Institute of Technology achieved a fill factor greater than $40 \%$ by using a matrix transform imager [95] Also in 2002, National Tsing-Hua University described an APS, with a fill factor of $55 \%$, made in $0.25 \mu \mathrm{m}$ technology [96]. In addition, this device has a high DR of $120 \mathrm{~dB}$, thanks to its innovative tuneable injection current compensation architecture and a voltage operation of $1.9 \mathrm{~V}$.

Finally, it is important to consider the downscaling effects, because small pixels mean lower light sensivity and dynamic range. Thus, conventional APS technology is limited. Nevertheless innovative architectures, ideas and technologies reaching a fill factor up to $90-100 \%$ have appeared: For instance, in 1997 Dierickx $[97,98]$ introduced a near $100 \%$ Fill Factor CMOS active pixel, which was 


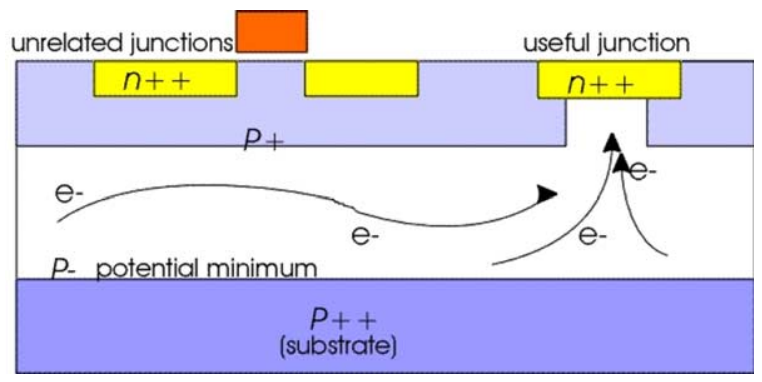

Fig. 7. Cross-section of Fill Factory's well pixel.

patented by FillFactory as High Fill Factor N-Well Pixel ${ }^{\circledR}$ (US Patent 6,225,670).

Photoelectrons are channeled by electrostatic barriers shielding them off the active pixel circuitry and substrate (see Fig. 7 left side), to the photodiode junction (see Fig. 7 right side). Virtually, all electrons diffuse down this drain, and as the diffusion time is short (typically 10-50 ns) negative effects like image lag must not be feared.

In addition, TFA Imagers [20-24] offer 100\% FF. The photodiode is placed on top of the ASIC. So the whole pixel area is available for the photodiode and there are no further layers obstructing the light penetration such as further metallization, polysilicon or dielectrics.

Increasing the photosensitivity of the photodetector is another thing to be taken into account in order to improve the quantum efficiency. M. Furumiya [99] reported in 2001 a high photosensitivity and no-crosstalk pixel technology for an APS by using a $0.35 \mu \mathrm{m}$ CMOS technology. A deep p-well photodiode, with a sensitivity improvement of $110 \%$ for $550 \mathrm{~nm}$ incident light, and an antireflective film to increase photosensitivity, consisting of $\mathrm{Si}_{3} \mathrm{~N}_{4}$ film, with a sensitivity improvement of $24 \%$ are used. Finally, it is possible to increase the sensitivity by the cascoding method, which allows shielding of the integrating capacitor from the parasitic junction capacitance of the photodiode. This can be done with shutter APS [44] or CTIA pixel [43].

\subsection{High performance (low power consumption and low voltage operation)}

One of the most important advantages of CMOS image sensors compared to CCDs is the lower power consumption. Therefore, CMOS image sensors are suitable for portable applications [31,100,101], among which, cellular phones, portable digital assistants (PDAs), and wireless security systems, etc.

A lot of research has been carried out on this topic. Low power camera-on-a-chip using CMOS APS technology began to be developed in 1995 by NASA at the Jet Propulsion Laboratory [102,103] and in 1998, the first CMOS APS fabricated using a high performance $1.8 \mathrm{~V}$, $0.25 \mu \mathrm{m}$ technology was introduced by Hon-Sum Philip Wong [104]. In that paper, the impact of the device scaling was studied, because no process modifications were made to the CMOS logic technology. In 1999, a CMOS imager with a power consumption of $250 \mathrm{~mW}$ [105] with an acquisition rate of 60 frames/s and a resolution of $1280 \times 720$ pixels was reported. This has been useful for large-format high-speed imaging applications such as industrial vision systems. In 2000, a $1.2 \mathrm{~V}$ micropower CMOS Active Pixel Image Sensor for portable applications was proposed [106]. In 2001, a low voltage hybrid Bulk/SOI CMOS APS was manufactured [107]. Also, in 2001, Nara Institute of Science and Technology reported a CMOS pixel circuit based on a pulse frequency modulation (PFM) technique [108]. This device reached a quite good performance (DR over $50 \mathrm{~dB}$ ) under very low operation voltage, less than $1 \mathrm{~V}$, and was very robust against noise due to its A/D converter. In 2003, a $176 \times 144$ CMOS APS with micropower consumption [109, 110] was reported, with a voltage operation of $1.5 \mathrm{~V}$ and power consumption of $550 \mu \mathrm{W}$. Thus, this amount enables the sensor to run using a watch battery.

Other novel designs have been introduced, like a CMOS imager with motion vector estimator for low power image compression [111], which was designed by Toyohashi University of Technology in 1999.

Someone states that APS will not function at $1.2 \mathrm{~V}$ or below [28]. However, CAPS [25,26,29,30] offer to work with a low voltage of $1 \mathrm{~V}$ or less using advanced technologies. They claim to obtain good performances [25,26,28-30]. Unlike APS, it must be stressed that CAPS are an alternative architecture which can be manufactured using top level technologies, below $0.25 \mu \mathrm{m}$, with great performance (see device scaling considerations). Thus, Low Voltage systems expect to continue downscaling by using CAPS, as an alternative to conventional APS.

\subsection{High speed imaging}

Acquiring high-speed images is becoming more and more important in some areas such as real time applications. Nevertheless CCD technology did not make enough progress in this aspect. During more than 3 decades CCDs have been developed and after spending millions of dollars, they reached 250 kilo-pixels with an acquisition speed of 1000 frames per second [19]. Comparatively, high speed CMOS sensor technology is just started, because the first high-speed sensors were introduced around 1998 [112,113] and they have reached already great results. In addition, high frame rates have been possible thanks to the CMOS downscaling. In conclusion, CMOS imagers appear to be a promising alternative to CCDs taken also into account other advantages such as less Blooming and Smearing effects.

\subsubsection{Needs and problem solving}

A typical CMOS APS contains three NMOS transistors in each pixel only. Therefore, a very compact implementation is possible although the sensor lacks of image data parallel acquisition, a feature often important in high-speed imaging. The alternative is a pixel that contains an analog 
memory called SNAP (Shuttered-Node Active Pixel). Another important issue is how the data is multiplexed into the output pads. Multiplexing of digital data is much simpler than passing off analog data, so ADC are needed. Another architectural feature that allows high-speed operation is pipelining [19].

A CMOS image sensor needs to fulfill all the necessary requirements in order to provide fast image acquision. No smear, no blooming and global electronic shutter are some of the most valuable characteristics needed [114]. In addition, low lag and snap-shot mode are preferable. Low lag is essential in order to capture rapidly changing scenes. The rolling shutter method is very common in CMOS imagers where the rows of pixels in the image sensor are sequentially reset, starting at the top of the image and proceeding row by row till the bottom. When this reset process has moved some distance down the image, the readout process begins: rows of pixels are read out sequentially as well, starting at the top of the image and proceeding row by row till the bottom in exactly the same fashion and at the same speed as the reset process. So, this device is not appropriate at high frame rates, because the scene can significantly change during the frame reading time. Therefore, a non-rolling shutter or snap-shot mode is necessary [115]. It is also necessary to acquire images in a very short time and using short integration times. This requires the image sensors to be equipped with synchronous shutter in order to avoid blur (see Fig. 8). For instance, images acquisition of fast-moving objects requires imagers with high photoresponsivity at short integration times, synchronous exposure, and high-speed parallel readout [116].

\subsubsection{Manufactured imagers}

Several designs have been reported since 1997. In 1998, a $128 \times 128$ snap-shot photogate CMOS imager in $0.5 \mu \mathrm{m}$ technology was implemented by Guang Yang [113]. It offered high speed (400 fps) and minimum exposure time $75 \mu \mathrm{m}$. It reproduces high quality, motion artifact-free images at high shutter-speeds ( $<75 \mu \mathrm{m}$ exposure), with low noise, unmeasurable image lag and excellent blooming protection.

Between 1998 and 2000, N. Stevanovic and M. Hillebrand $[6,116,117,114]$ reported a high speed
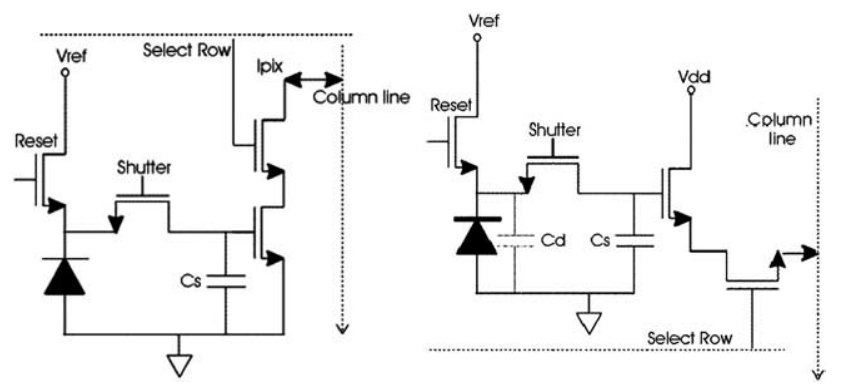

Fig. 8. Photodiode-type shutter APS schematics.

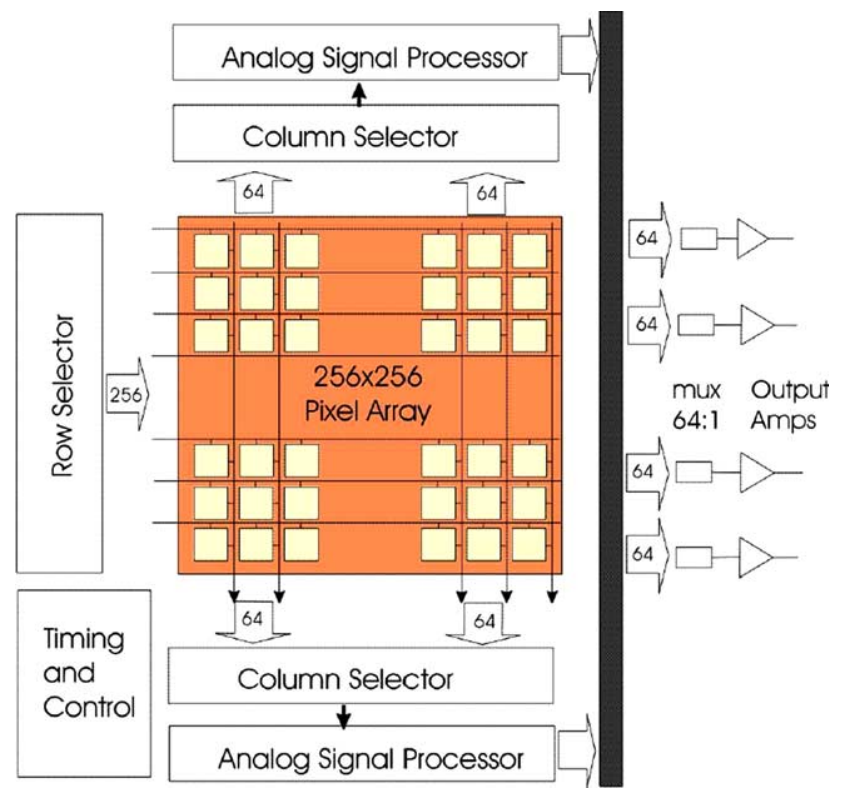

Fig. 9. Architecture of the high speed CMOS imager.

CMOS camera (see Fig. 9). It was able to acquire more than 1000 frame/s using a global shutter in each sensor cell. The integration time in synchronous exposure was variable between $1 \mu \mathrm{s}$ and 150 instead of previous CMOS implementations, which had around 500 frames/s at integration times ranging from 75 to $200 \mu \mathrm{m}[42,113,112]$. So it offered a compact, portable, and low power $(320 \mathrm{~mW})$ solution for high speed video systems and had a resolution of $256 \times 256$ pixels.

DALSA Inc. Waterloo reported a VGA CMOS imager [115] in 2001, which can capture images at 1600 frames per second (see Fig. 10). Furthermore, it has exposure control functionality, antiblooming capability and a non-rolling shutter architecture to implement snap-shot image capture mode.

Also in 2001, S. Yoshimura, T. Sugiyama, K. Yonemoto and $\mathrm{K}$. Ueda reported a $48 \mathrm{kframe} / \mathrm{s}$ CMOS Image sensor for

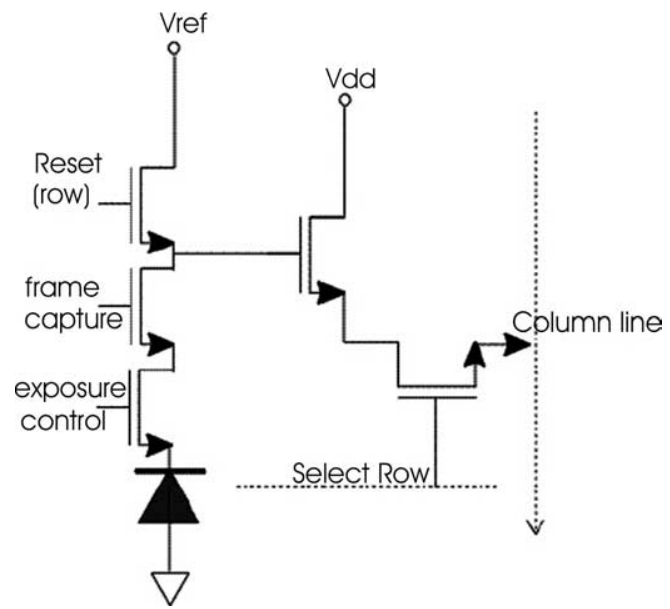

Fig. 10. Pixel of a DALSA VGA CMOS imager. 
Real-time 3D Sensing and Motion Detection [118]. It had an array of $192 \times 124$ pixels, depth resolution of $500 \mu \mathrm{m}$, fast motion detection and $12 \mathrm{~b}$ digital image output resolution.

E. Fossum and A. Krymski introduced first a $1280 \times 720$ pixel at $60 \mathrm{fps}(60 \mathrm{Mpixel} / \mathrm{s})$, then reported a $1024 \times 1024$ pixel at $500 \mathrm{fps}(500 \mathrm{Mpixel} / \mathrm{s})$. They continued enhancing their design, when in 2003 they presented [119] a high speed, $240 \mathrm{fps}$, $4.1 \mathrm{Mpixel}(2352 \times 1728)$ CMOS sensor $(>$ $800 \mathrm{Mpixels} / \mathrm{s}$ ) with on-chip parallel 10-b analog-to-digital converters (ADCs) and power dissipation of less than $700 \mathrm{~mW}$. Besides this, in 2003, a High-responsivity 9V/Lux-s, high speed $5000 \mathrm{fps}$ at full $512 \times 512$ resolution CMOS sensor was manufactured [44]. The sensor was designed for a $0.35 \mu \mathrm{m}$ process and consisted of a five transistor pixel to provide a true parallel shutter.

\subsubsection{High speed market}

High speed imaging systems are suitable for automotive applications such as occupancy detection, precrash sensing, collision avoidance, surveillance, crash test observation, or airbag control. For instance, a smart airbag solution based on a high speed camera system was designed by Fraunhofer Institute of Microelectronic Circuits and Systems [120]. The system continuously monitors the seats and quickly determines the occupancy status and passenger's position and size before the airbag is blasted. Another application is smart image sensor for real-time. For instance Yosuke Oike reported in 2003 a smart image sensor for real-time and high-resolution 3D measurement [121]. It does not only have enough high frame rate for real-time 3D measurement, but also high pixel resolution owing to a small pixel circuit and high subpixel accuracy due to gravity center calculation using a light intensity profile measurement trick. Finally, an application for high-speed video systems, for fast moving objects or for machinery vision is also suitable.

\subsection{Low noise sensors}

CMOS Image sensors suffer from several noise sources. These set the fundamental limits on image sensor performance, especially under low illumination and in video applications. Therefore, it is important to have an overview of all of them [80]. The noise sources in CMOS Imagers can be divided in Temporal Noise [81] and Fixed Pattern Noise (FPN) [43].

In fact, FPN is one of the major CMOS imager's disadvantages. Thus, a lot of research has been done in order to minimise FPN.

Many researchers have designed FPN-reduction circuits. For instance, Correlated Double Sampling (CDS) is one of the most suitable for suppressing FPN [50,76]. Fig. 11 shows a typical schematic of a CDS circuit. CDS technique consists of taking two samples from a signal, which are closely spaced in time. Then, the first signal is substratcted from the second one, hence, removing the low-frequency noise. Sampling occurs twice: first after reset and last after

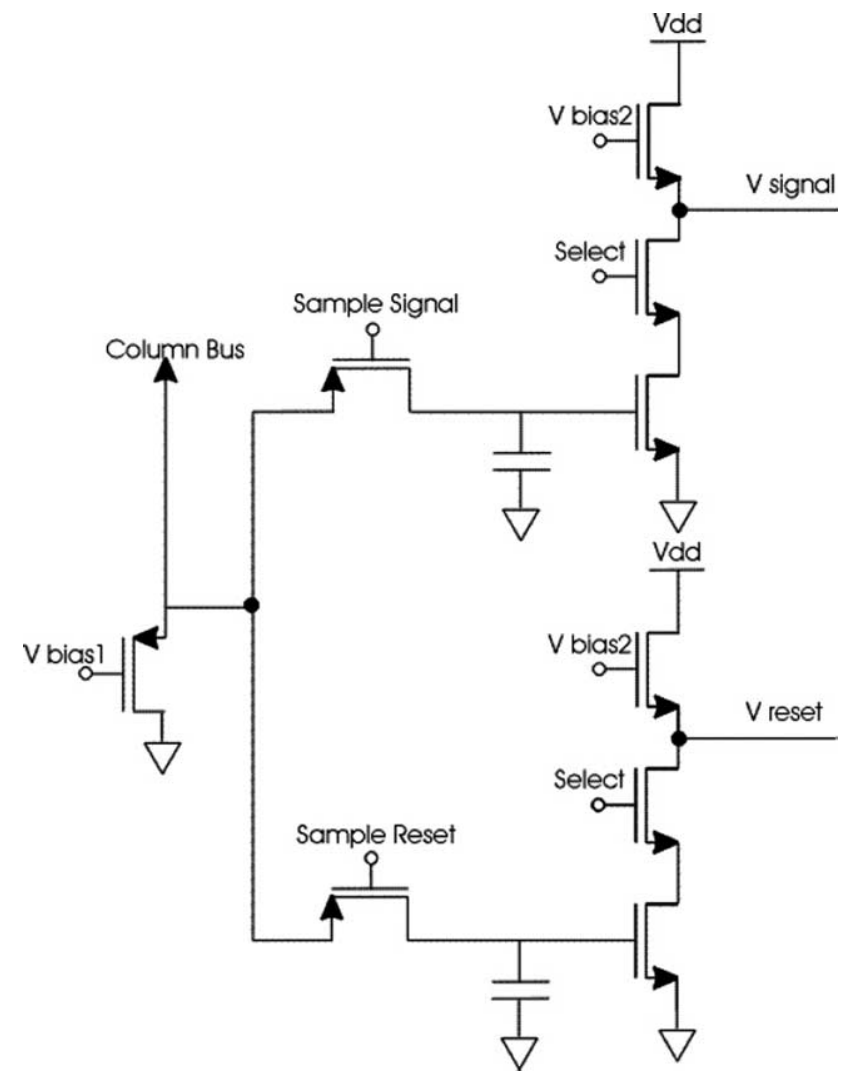

Fig. 11. Schematic diagram of the correlated double sampling circuit. There is one such circuit for every column.

integrating the signal charge. The subtraction removes the reset noise and dc offset from the signal charge. The two values are then used as differential signals in further stages like programmable gain amplifiers (PGA) or ADC. Most of them are placed below each column of pixels (see Fig. 12a). However, CDS reduces the fixed pattern noise to a large extent, a component of the FPN due to mismatch in the CDS circuits at each column introduces column-FPN, which should be also removed. For instance, K. Yonemoto and H. Sumi proposed [50] that FPN reduction should be performed in a CDS circuit (see Fig. 12b), in order to avoid this column-FPN caused by CDS circuits. On the other hand, although the dark current variation of photodiodes appears as FPN in the output signal of a CMOS image sensor, which resembles FPN caused by threshold variation of transistors in pixel circuits, the dark current noise cannot be suppressed with CDS circuits. This is because the dark current does not appear in the reset level, but only in the signal level of the pixel signal. Therefore, the dark current of the photodiode itself should be reduced.

One way of reducing the dark current is to employ a pinned photodiode [122]. Another method reported by K. Yonemoto and H. Sumi in 2000 is a pinned photodiode, in the form of hole accumulation diode (HAD) [50]. They achieved a reduction of the dark current to $150 \mathrm{pA} / \mathrm{cm}^{2}$ instead of $6 \mathrm{nA} / \mathrm{cm}^{2}$ of a pn-photodiode. As a result, the dark 
(a)

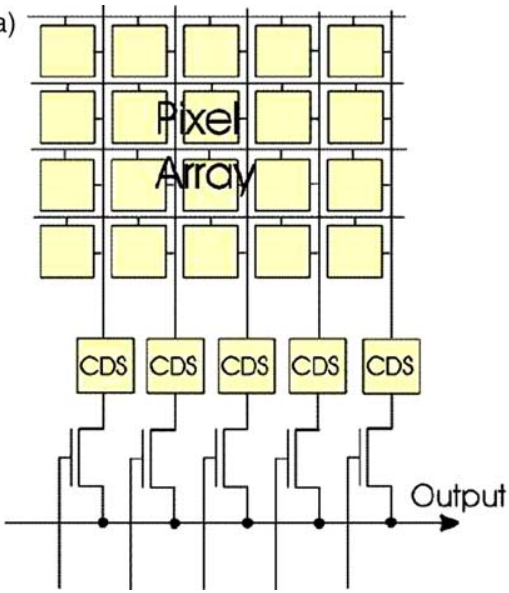

(b)

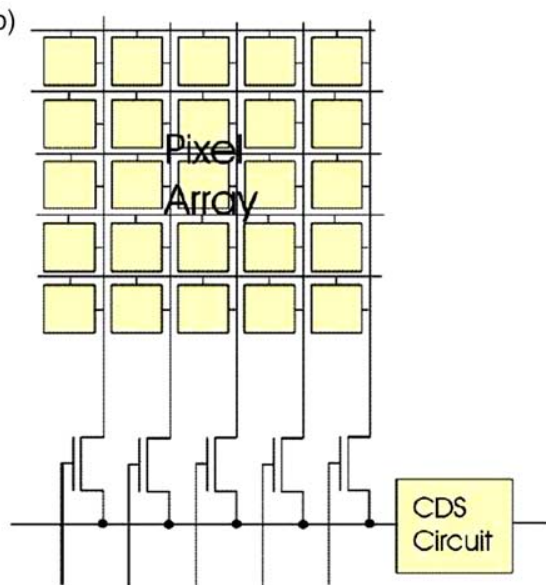

Fig. 12. (a) CMOS image sensor with column CDS circuit, (b) CMOS image sensor with proposed FPN-reduction scheme.

current variation at the output of the CMOS image sensor was $0.19 \mathrm{mV}$ and the period of readout operation was about $20 \mathrm{~ns}$ at 30 frames/s. Two years later, K. Yonemoto and H. Sumi carried out [49] a numerical analysis of this CMOS Image sensor with a simple FPN reduction technology. They showed that the low-input-voltage I-V converter with a current-mirror circuit improves the amplification factor and linearity of the pixel circuit. In a five-transistor pixel circuit, the threshold voltage of the $\mathrm{X}-\mathrm{Y}$ addressing transistor affects the amplitude and the level of the readout pulse. An analysis of the mechanism of the $\mathrm{X}-\mathrm{Y}$ addressing transistor showed the basic concept behind the selection of the threshold voltage. An L-shaped readout gate for a pinned photodiode was compared with a straight readout gate, and was proved to be adequate for rapid charge transfer.

Another circuit to suppress FPN peak-to-peak to $0.15 \%$ of saturation level is Delta-difference sampling (DDS) [123].

On the other hand, B. Fowler [43] proposed a new APS, called Capacitive Transimpedance Amplifier (CTIA). CTIA $A P S$ that can achieve low FPN by using a divider circuit with switched capacitor voltage feedback. Besides this, the high gain and low read noise are advantages of using a CTIA as well (See Fig. 5h and g).

Moreover, other readout methods can also offer an improvement in order to suppress FPN. For instance, R\&D Headquarters from Minolta Co. and Gazoh System Kaihatsu reported a CMOS APS with transversal readout architecture that eliminates the vertically striped FPN $[73,75]$. The possibility of high frame rate using a multiport structure was also demonstrated. In addition, the Photonics and Sensors Group of the Cambridge University suggested, in 2002 [74], a new readout circuit for a CMOS APS, which removes the FPN and reduces signal degradation while offering an increase in readout speed compared to the conventional approach.

As outlined before, CDS can not suppress the dark current noise, although it is a FPN's source. Thus, decreasing the dark current to suppress FPN has been an aim. Dark current (offset error) is the signal charge that the pixel collects in the absence of light divided by the integration time. Dark current is temperature-sensitive and typically normalised by area. Photobit Technology Corporation and Tokyo institute of Technology reported a low dark current stacked CMOS APS for charged particle detection [124,125]. A use of a p-MOSFET transistor for readout reduces the hot carrier effect; thereby the dark current within the low temperature region is greatly decreased. It also improves noise reading performance due to its lower flicker noise compared to n-MOSFETs'.

Thanks to the improvement of the noise performance, CMOS Image Sensors for Low light level applications are possible [18]. In 2000, a CDS noise analysis of readout circuits used in CMOS APS for low light levels was carried out [17]. In 2001, different pixel architectures were studied in order to increase the sensitivity and reduce the spatial (FPN) and temporal noise [16]. This study demonstrates that the N-well photodiode is the best light sensor, either for its parasitic capacitance value, for its quantum efficiency, or for its dark current. However, the design rules required by this photodiode (a wide space must be kept between $\mathrm{N}$ well and MOS transistors) limit their use in CMOS imagers. On the other hand, a new pixel architecture was also introduced. This architecture reduces kTC or reset noise and FPN. Therefore, this architecture is ideal for applications requiring very high sensitivity and low noise, which is necessary for low light level sensing.

Complete reset of the photodiode is needed in order to remove kTC or reset noise and decrease the lag effect. Note that the source of image lag in CMOS imagers is different from the source of image lag in CCDs. In CCDs, image lag is caused by incomplete charge transfer. This can be eliminated using a pinned photodiode. On the other hand, CMOS image lag is due to incomplete reset so, in 2001, H. Tian [81] reported a new reset method, which alleviates the lag without increasing the reset noise. The reset transistor gate is overdriven.

Finally, CMOS APS still has readout-noise problems because of irregular gain from mismatched transistor thresholds. 


\section{Applications}

As highlighted before, improvement of CMOS image sensors has opened up new application areas [45]. Therefore, CMOS imagers are very suitable for Space, Automotive, Medical, Digital photography and 3D applications. Furthermore, there are more specific applications such as portable devices [100,101,31], security, industrial vision $[126,127]$, consumer electronics, imaging phones, astronomy, surveillance [128], robotics and machine vision, guidance and navigation (e.g., stereovision [129]), computer inputs, etc.

\subsection{Space applications}

CMOS Imagers are widely used in the space environment for a varied range of applications [130,131]. These applications include robotic and navigation cameras, imagers for astronomy and earth observation, star trackers [132], tracking sensors in satellite constellations, lander and rover imagers, X-ray satellite missions [133,134], etc. Moreover, CMOS imagers are known to be tolerant to radiation, although true radiation tolerance can only be obtained using specific methods. Thus, there is a huge interest in radiation-tolerant imaging systems $[32-39,135$, 136]

\subsection{Automotive applications}

There are a lot of applications [137] in the automotive field like occupancy detection, airbag control, precrash sensing, collision avoidance, surveillance, crash test observation, etc. Another one is a smart airbag solution based on a high speed camera system [120]. This system continuously monitors the seats and quickly determines the occupancy status and passenger's position and size before the airbag is blasted. Moreover, IR-vision systems for foggy and night driving conditions are also addressable.

\subsection{Medical applications}

Medical or Biomedical systems based on CMOS imagers have been successfully developed [138]. For instance, Microelectronic components for a retina-implant system that will provide visual sensations to patients suffering from photoreceptor degeneration was reported by M. Schwarz in 1999 [139]. On the other hand, the digitisation of medical images, especially in radiology, has been another demand in recent years. Ho Kyung Kim proposed an X-ray imaging system with large FOV (field-of-view) using CMOS image sensors [140]. S. Wook Lee reported a 3-D Xray microtomographic system [141] in 2001. It makes possible to see the internal structure of small objects in a nondestructive way. Finally, P. Lechner developed an APS for $\mathrm{X}$-ray imaging Spectroscopy in 2001 [142].

\subsection{Digital photography}

A CMOS image sensor integrating the sensor itself and the digital control functions on a single chip was reported [1]. This demonstrates the viability of producing a camera-on-a-chip suitable for commercial and scientific applications $[143,144]$. Besides, cameras with nearly noisefree pictures [145] and low power consumption have been developed. In 1998, Toshiba Corp. reported a $3.7 \times 3.7 \mu \mathrm{m}^{2}$ square pixel CMOS image sensor [146] for Digital Still Camera applications with high performance.

\subsection{1. $3 D$ range imaging applications}

3D range imaging systems are more and more required due to the fact that 3D images acquisition is important in various sectors such as home, public and industrial domains. Furthermore, improvements in speed and resolution performance have opened up the possibility to obtain real time systems.3D range imaging system, also called 3D digitiser or Range finder, is a system capable to acquire range or depth information. These devices grab 'range images' or ' images', which are dense arrays of values related to the distance of the scene to a known point or plane [147]. Currently, there are some special 3D measurement methods available for scene reconstruction. These techniques rely on triangulation (see Fig. 13), time-of-flight (TOF) measurements or interferometry, etc. [148] The range of possible applications is wide. For instance, obtaining 3D Models from Range Scans [149], Space monitoring and surveillance [118], safety and security [148,120], Real time Sensing and Motion detection [118], Inspection [150], 3D X-ray imaging [141], Robot vision [151], etc.

\subsection{Other applications}

There are more suitable applications, such as portable applications [100,101,31], security, industrial vision (e.g., Imager with focal plane edge detection [127]), consumer electronics, surveillance devices [128], Smart vision system on-a-chip [143,144,129,152], Robotics and machine vision,

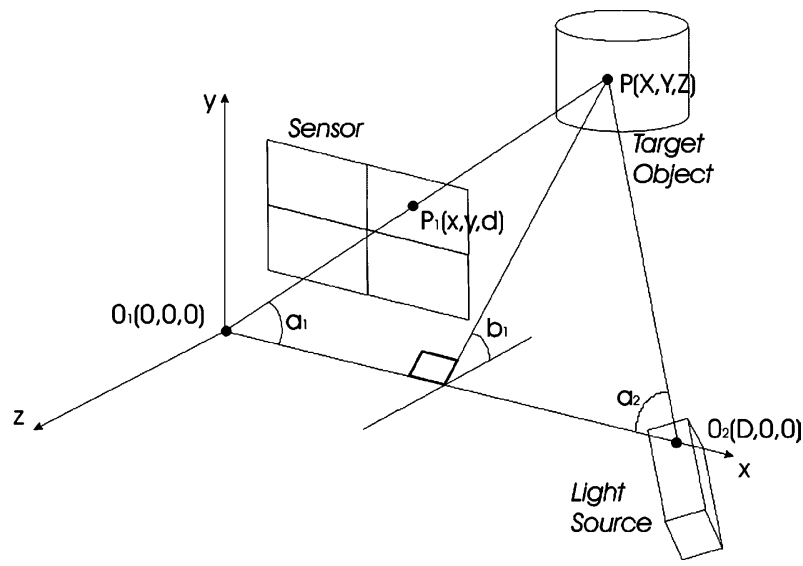

Fig. 13. 3D measurement system based on triangulation. 
guidance and navigation (e.g., stereovision [129]), video phones, computer inputs, for charged particle imaging [124, 125] such as ion and electron imaging, IR-vision applications, low light level applications [16-18], electrostatic sensing, instrumentation, imaging phones, astronomy and low-end professional cameras.

\section{Conclusions}

A review of the most important advances in the field of CMOS image sensors has been carried out. These advantages have been mainly focused on fields such as sensitivity, low noise, low power consumption, low voltage operation, high-speed imaging and good dynamic range. This paper demonstrates that CMOS imagers are competitive with CCDs in many application areas, such as security, consumer digital cameras, automotive, computer video, imaging phones, etc. CMOS imagers will replace CCD devices in some cases, because of its low cost, low power consumption, integration capability, etc. Nevertheless, CCD technology will continue as predominant in high performance systems, such as medical imaging, astronomy, low-end professional cameras, etc. because of its better image quality. To sum up, State-of-the-art of CMOS image sensors has been provided.

\section{References}

[1] E. Fossum, Cmos image sensors: electronic camera-on-a-chip, IEEE transactions on electron devices 44 (10) (1997).

[2] S. Kempainen, Cmos image sensors:eclipsing ccds in visual information?, EDN 42 (21) (1997) 101-102 [See also pp. 105-6, $108,110]$.

[3] C.-H. Chen, H.-J. Tsai, K.-S. Huang, and H.-T. Liu, Study for cross contamination between cmos image sensor and ic product, in 2001 IEEE/SEMI Advanced Semiconductor Manufacturing Conference, 2001, pp. 121-123.

[4] H.-S.P. Wong, Cmos image sensors-recent advances and device scaling considerations, in International Electron Devices Meeting 1997. IEDM, 1997, pp. 201-204.

[5] A. Theuwissen, Ccd or cmos image sensors for consumer digital still photography? in International Symposium on VLSI Technology, Systems, and Applications, 2001. Proceedings of Technical Papers, 2001, pp. 168-171.

[6] M. Hillebrand, N. Stevanovic, B. Hosticka, J. Conde, A. Teuner, and M. Schwarz, High speed camera system using a cmos image sensor, in Proceedings of the IEEE Intelligent Vehicles Symposium 2000. 2000, pp. 656-61.

[7] W.S. Boyle, G. Smith, Charge-coupled semiconductor devices, Bell System Techenics of Journal 49 (1970) 587-593.

[8] J. Zarnowski, M. Pace, M. Joyner, Active-pixel cmos sensor improve their image, Laser Focus World. PennWell Plublishing 35 (7) (1999) $111-114$.

[9] I. Shcherback, O. Yadid-Pecht, Photoresponse analysis and pixel shape optimization for cmos active pixel sensors, IEEE Transactions on Electron Devices 50 (2003) 12-18.

[10] W. Zhang, M. Chan, H. Wang, and P. Ko, Building hybrid active pixels for cmos imager on soi substrate, in SOI Conference. Proceedings. IEEE International, 1999, pp. 102-103.
[11] Y. Malinovich, Ultra-high resolution cmos image sensors, Electronic Product Design. IML Techpress 20 (7) (1999) 19-20.

[12] Z. Zhou, B. Pain, and E. Fossum, A cmos imager with on-chip variable resolution for light-adaptive imaging, in IEEE International Solid-State Circuits Conference. 45th ISSCC, Feb. 1998, pp. 174-175, 433 .

[13] J. Coulombe, M. Sawan, and C. Wang, "Variable resolution cmos current mode active pixel sensor, in IEEE International Symposium on Circuits and Systems. Proceedings. ISCAS 2000 Geneva., vol. 2, 2000, pp. 293-296.

[14] Y. Audet and G. Chapman, Design of a self-correcting active pixel sensor, in IEEE International Symposium on Defect and Fault Tolerance in VLSI Systems, 2001. Proceedings., 2001, pp. 18-26.

[15] I. Koren, G. Chapman, and Z. Koren, A self-correcting active pixel camera, in IEEE International Symposium on Defect and Fault Tolerance in VLSI Systems, 2000. Proceedings., 2000, pp. 56-64.

[16] J. Goy, B. Courtois, J. Karam, F. Pressecq, Design of an aps cmos image sensor for low light level applications using standard cmos technology Analog Integrated Circuits and Signal Processing, vol. 29, Kluwer Academic Publishers, Dordrecht, 2001. pp. 95-104.

[17] Y. Degerli, F. Lavernhe, P. Magnan, J. Farre, Analysis and reduction of signal readout circuitry temporal noise in cmos image sensors for low-light levels, IEEE Transactions on Electron Devices 47 (5) (2000) 949-962.

[18] D. Croft, Cmos image sensors compete for low-light tasks, Laser Focus World. PennWell Publishing 35 (1) (1999) 135-136 [See alspo pp. 138-40].

[19] E. Fossum and A. Krymski, High speed cmos imaging. in ElectronicEnhanced Optics, Optical Sensing in Semiconductor Manufacturing, Electro-Optics in Space, Broadband Optical Networks. Digest of the LEOS Summer Topical Meetings, July 2000, pp. I3-I4.

[20] T. Lule, S. Benthien, H. Keller, F. Mutze, P. Rieve, K. Seibel, M. Sommer, M. Bohm, Sensitivity of cmos based imagers and scaling perspectives, IEEE transactions on electron devices 47 (2000) 2110-2122.

[21] T. Lule, M. Wagner, M. Verhoeven, H. Keller, M. Bohm, 100000pixel, 120-db imager in tfa technology, IEEE Journal of Solid-State Circuits 35 (2000) 732-739.

[22] T. Lule, H. Keller, M. Wagner, and M. Bohm, 100.000 pixel $120 \mathrm{db}$ imager in tfa-technology, in Symposium on VLSI Circuits, 1999. Digest of Technical Papers, June 1999, pp. 133-136.

[23] T. Lule, B. Schneider, M. Bohm, Design and fabrication of a highdynamicrange image sensor in tfa technology, IEEE Journal of Solid-State Circuits 34 (1999) 704-711.

[24] B. Schneider, H. Fischer, S. Benthien, H. Keller, T. Lule, P. Rieve, M. Sommer, J. Schulte, and M. Bohm, Tfa image sensors: from the one transistor cell to a locally adaptive high dynamic range sensor, in Electron Devices Meeting, 1997. Technical Digest., International, 1997, pp. 209-212.

[25] C. Xu, W.-H. Ki, M. Chan, A low-voltage cmos complementary active pixel sensor (caps) fabricated using a 0.25 (m cmos technology, IEEE Electron Device Letters 23 (2002) 398-400.

[26] C. Xu, W. Zhang, W.-H. Ki, and M. Chan, A highly integrated cmos image sensor architecture for low voltage applications with deep submicron process, in IEEE International Symposium on Circuits and Systems. ISCAS 2002., vol. 3, 2002, pp. $699-702$.

[27] C. Shen, C. Xu, Weiquan, W.R. Huang, and M. Chan, Low voltage cmos active pixel sensor design methodology with device scaling considerations, in IEEE Hong Kong Electron Devices Meeting, June 2001, pp. 21-24.

[28] C. Xu, W. Zhang, and M. Chan, A $1.0 \mathrm{v}$ vdd cmos active pixel image sensor with complementary pixel architecture fabricated with a $0.25 \mathrm{~m}$ cmos process, in IEEE International Solid-State Circuits Conference. ISSCC, vol. 1, 2002, pp. 44-443. 
[29] -, A 1.0 vdd cmos active pixel image sensor with complementary pixel architecture fabricated with a $0.25 \mathrm{~m}$ cmos process, in IEEE International Solid-State Circuits Conference. ISSCC, vol. 2, 2002, pp. 28-385.

[30] C. Xu, W. Zhang, W.-H. Ki, M. Chan, A 1.0-v vdd cmos active-pixel sensor with complementary pixel architecture and pulsewidth modulation fabricated with a 0.25 ( $\mathrm{m}$ cmos process, IEEE Journal of Solid-State Circuits 37 (2002) 1853-1859.

[31] C. Xu and M. Chan, The approach to rail-to-rail cmos active pixel sensor for portable applications, in Proceedings of IEEE Region 10 International Conference on Electrical and Electronic Technology. TENCON, vol. 2, 2001, pp. 834-837.

[32] E.-S. Eid, S. Ay, and E. Fossum, Design of radiation tolerant cmos aps system-on-a chip image sensors in Aerospace Conference Proceedings, vol. 4, 2002, pp. 2005-2011.

[33] E.-S. Eid, T. Chan, E. Fossum, R. Tsai, R. Spagnuolo, and J. Deily, Design of radiation hard cmos aps image sensors in $0.35 \mathrm{~m} \mathrm{cmos}$ standard process, in Proceedings of the SPIE-The International Society for Optical Engineering, vol. 4306, 2002, pp. 50-9.

[34] J. Bogaerts, B. Dierickx, R Mertens, Random telegraph signals in a radiationhardened cmos active pixel sensor, IEEE Transactions on Nuclear Science 49 (2002) 249-257.

[35] J. Bogaerts, B. Dierickx, G. Meynants, D. Uwaerts, Total dose and displacement damage effects in a radiation-hardened cmos aps, IEEE Transactions on Electron Device 50 (84) (2003) 90.

[36] J. Bogaerts, B. Dierickx, R. Mertens, Enhanced dark current generation in proton-irradiated cmos active pixel sensors, IEEE Transactions on nuclear science 49 (2002) 1513-1521.

[37] M. Cohen and J. David, Radiation effects on active pixel sensors, in Fifth European Conference on Radiation and Its Effects on Components and Systems. ADECS 99., 1999, pp. 450-456.

[38] -, "Radiation-induced dark current in cmos active pixel sensors," IEEE Transactions on Nuclear Science, vol. 47, pp. 2485-2491, 2000.

[39] G. Hopkinson, Radiation effects in a cmos active pixel sensor, IEEE Transactions on Nuclear Science 47 (2000) 2480-2485.

[40] C.-C. Wang and C. Sodini,The effect of hot carriers on the operation of cmos active pixel sensors, in International Electron Devices Meeting, 2001. IEDM Technical Digest., 2001, pp. 24.5.1-.5.4.

[41] C. Seibold. (2002) Comparison of cmos and ccd image sensor technologies. [Online]. Available: http://eng.oregonstate.edu/ millerst/ece $44 x$.

[42] A. Krymski, D. Blerkom, A. Andersson, N. Bock, B. Mansoorian, and E.R. Fossum,A high speed, 500 frame/s, 1024x1024 cmos active pixel sensor, in IEEE Symposium on VLSI Circuits Digest of Technical Papers, 1999, pp. 137 -138.

[43] B. Fowler, J. Balicki, D. How, and M. Godfrey, Low fpn high gain capacitive transimpedance amplifier for low noise cmos image sensors, in Proceedings of the SPIE. The International Society for Optical Engineering, vol. 4306, 2001, pp. 68-77.

[44] A.I. Krymski, N. Tu, A 9-v/lux-s 5000-frame/s $512 \times 512 \mathrm{cmos}$ sensor, IEEE Transactions on electron devices 50 (1) (2003) $136-143$.

[45] C.H. Small, "Lower costs open new application areas for cmos image sensors, Computer Design, International Edition, PennWell Publishing 37 (4) (1998) 37-40 [See also pp. 42-43].

[46] H.-S. Wong;, Technology and device scaling considerations for cmos imagers, IEEE Transactions on Electron Devices 43 (1996) 2131-2142.

[47] S. Mendis, S. Kemeny, R. Gee, B. Pain, C. Staller, Q. Kim, E. Fossum, Cmos active pixel image sensor for highly integrated imaging systems, IEEE Journal of Solid-State Circuits 32 (1997) 187-197.

[48] Y. Muramatsu, S. Kurosawa, M. Furumiya, H. Ohkubo, Y. Nakashiba, A signalprocessing cmos image sensor using a simple analog operation, IEEE Journal of Solid-State Circuits 38 (2003) $101-106$
[49] K Yonemoto, H Sumi, A numerical analysis of a cmos image sensor with a simple fixed-pattern-noise-reduction technology, IEEE Transactions on Electron Devices 49 (2002) 746-753.

[50] A cmos image sensor with a simple fixed-pattern-noise-reduction technology and a hole accumulation diode, IEEE Journal of SolidState Circuits, vol. 35, pp. 2038-43, 2000.

[51] J.-S. Ho, M.-C. Chiang, H.-M. Cheng, T.-P. Lin, and M.-J. Kao, A new design for a $1280 \times 1024$ digital cmos image sensor with enhanced sensitivity, dynamic range and fpn, in 1999 International Symposium on VLSI Technology, Systems, and Applications., 1999, pp. 235-238.

[52] R. Nair and K. Raj, Signal processing in cmos image sensors, in IEEE Workshop on Signal Processing Systems. SIPS 2000, 2000, pp. 801-810.

[53] B. Sekerkiran, U. Cilingiroglu, A cmos k-winners-take-all circuit with o(n) complexity, IEEE Transactions on Circuits and Systems, II, Analog and Digital Signal Processing 46 (1) (1999) 1-5.

[54] A. Demosthenous, S. Smedley, J. Taylor, A cmos analog winnertake-all network for large-scale applications, IEEE Transactions on Circuits and Systems I: Fundamental Theory and Applications 45 (3) (1998) 300-304.

[55] J.-C. Yen, J.-I. Guo, H.-C. Chen, A new k-winners-take-all neural network and its array architecture, IEEE Transactions on Neural Networks 9 (5) (1998) 901-912.

[56] N. Donckers, C. Dualibe, and M. Verleysen. (1999) Design of complementary low-power cmos architectures for looser-take-all and winner-take-all. [Online]. Available: citeseer.nj.nec.com/donckers99design.html.

[57] D.Y. Aksin, A high-precision high-resolution wta-max circuit of o(n) complexity, IEEE Transactions on Circuits and Systems 49 (1) (2002) 48-53.

[58] G. Indiveri, P. Oswald, and J. Kramer, An adaptive visual tracking sensor with a hysteretic winner-take-all network, in IEEE International Symposium on Circuits and Systems. ISCAS 2002, vol. 2, 2002, pp. II-324-II-327.

[59] F. Blais, M. Lecavalier, and J. Bisson, Real-time processing and validation of optical ranging in a cluttered enviroment, in The 7th International Conference on Signal Processing Applications \& Technology. ICSPAT'96, Oct. 1996.

[60] Z. Zhang and V. Prinet, A rogh-to-fine satellite image registration method with subpixel accuracy, in International Conference on Image Processing, vol. 3, 2002, pp. III-385 -III-388.

[61] L. Gonzo, M. Gottardi, F. Comper, A. Simoni, J.-A. Beraldin, F. Blais, M. Rioux, and J. Domey. Smart sensors for 3d digitization. [Online]. Available: http://www.vit.iit.nrc.ca/References/NRC44934.pdf.

[62] F. Eugenio, F. Marques, and J. Marcello, Pixel and sub-pixel accuracy in satellite image georeferencing using an automatic contour matching approach, in International Conference on Image Processing, vol. 1, 2001, pp. 822-825.

[63] H. Kwon, S.Z. Der, and N.M. Nasrabadi. (2002) Subpixel target detection for hyperspectral images using ica-based feature extraction. [Online]. Available: http://www.asc2002.com/manuscripts/J/ JO-03.PDF.

[64] S.S. Abeysekera, An efficient hilbert transform interpolation algorithm for peak position estimation, in Proceedings of the 11th IEEE Signal Processing Workshop on Statistical Signal Processing, 2001, pp. 417-420.

[65] J. Xu, Z.P. Fang, A.A. Malcolm, H. Wang, "Camera calibration for 3-d measurement with micron level accuracy," in 5th (2001) 2001.

[66] P.C. Yu, S.J. Decaer, H.-S. Lee, C.G. Sodini, J. John, L. Wyatt, Cmos resistive fuses for image smoothing and segmentation, IEEE Journal of Solid-State Circuits 27 (4) (1992) 545.

[67] S.-B. Park, A. Teuner, and B. Hosticaka, A motion detection system based on a cmos photo sensor array, in International Conference on Image Processing, 1998. 
[68] S.-M. Sohn, M.G. Kim, S. Kim;, “A cmos image sensor (cis) with low power motion detection for security camera applications," in IEEE International Conference on Consumer Electronics, ICCE. 2003, June 2003, p. 250 (2003) 251.

[69] Y. Muramatsu, S. Kurosawa, M. Furumiya, H. Ohkubo, Y. Nakashiba, A signal-processing cmos image sensor using a simple analog operation, IEEE Journal of Solid-State Circuits 38 (1) (2003) 101-106.

[70] S. Kemeny, B. Pain, L. Matthies, E. Fossum, Multiresolution image sensor, IEEE Transactions on Circuits and Systems for Video Technology 7 (1997) 575-583.

[71] S. Kawahito, Y. Tadokoro, A. Matsuzawa, "Cmos image sensors with video compression," in Proceedings of the ASP-DAC '98. Asia and South Pacific Design Automation Conference., Feb., p. 595 (1998) 600.

[72] Y.-C. Shih and C.-Y. Wu, The design of high-performance $128 \times$ $128 \mathrm{cmos}$ image sensors using new current-readout techniques, in IEEE International Symposium on Circuits and Systems. ISCAS '99, vol. 5, May 1999, pp. 168-171.

[73] S. Miyatake, M. Miyamoto, K. Ishida, T. Morimoto, Y. Masaki, H. Tanabe, Transveral-readout architecture for cmos active pixel image sensors, IEEE Transactions on Electron Devices 50 (2003) 121-129.

[74] T. Kwok, J.J. Zhong, T. Wilkinson, W.A. Crossland, Readout circuit for cmos active pixel image sensor, IEEE Electronics Letters 38 (2002) 317-318.

[75] S. Miyatake, K. Ishida, T. Morimoto, Y. Masaki, and H. Tanabe, Transversal-readout cmos active pixel image sensor, in Proceedings of the SPIE - The International Society for Optical Engineering, vol. 4306, 2001, pp. 128-136.

[76] Y. Degerli, F. Lavernhe, P. Magnan, P.J. Farre, Column readout circuit with global charge amplifier for cmos aps imagers, IEEE Electronics letters 36 (17) (2000) 1457-1459.

[77] T. Watabe, M. Goto, H. Ohtake, H. Maruyama, M. Abe, K. Tanioka, N. Egami, New signal readout method for ultrahighsensitivity cmos image sensor, IEEE Transactions on Electron Devices 50 (2003) 63-69.

[78] T. Watabe, M. Goto, H. Ohtake, H. Maruyama, and K. Tanioka, A new readout circuit for an ultra high sensitivity cmos image sensor," in International Conference on Consumer Electronics. ICCE. 2002, 2002, pp. 42-43.

[79] C.-C. Hsieh, C.-Y. Wu, F.-W. Jih, T.-P. Sun, Focal-plane-arrays and cmos readout techniques of infrared imaging systems, IEEE Transactions on Circuits and Systems for Video Technology 7 (1997) 594-605.

[80] HPComponentsGroup. (1998) Noise sources in cmos image sensors. [Online]. Available: http://www.stw.tuilmenau.de/ ff/beruf_cc/cmos/cmos_noise.pdf.

[81] H. Tian, B. Fowler, A.E. Gamal, Analysis of temporal noise in cmos photodiode active pixel sensor, IEEE Journal of Solid-State Circuits 36 (1) (2001) 92-101.

[82] O. Schrey, J. Huppertz, G. Filimonovic, A. Bussmann, W. Brockherde, B. Hosticka, A $1 \mathrm{k} \times 1 \mathrm{k}$ high dynamic range cmos image sensor with on-chip programmable region-of-interest readout, IEEE Journal of Solid-State Circuits 37 (2002) 911-915.

[83] M. Schanz, C. Nitta, A. Bussmann, B.J. Hosticka, R.K Wertheimer, A high-dynamic-range cmos image sensor for automotive applications, IEEE Journal of Solid-State Circuits 35 (2000) 932-938.

[84] M. Loose, K. Meier, and J. Schemmel, Cmos image sensor with logarithmic response and self calibrating fixed pattern noise correction, in Proceedings of the SPIE - The International Society for Optical Engineering, vol. 3410, 1998, pp. 117-27.

[85] R. Hauschild, M. Hillebrand, B.J. Hosticka, J. Huppertz, T. Kneip and M. Schwarz, A cmos image sensor with local brightness adaptation and high intrascene dynamic range, in Proceedings of the 24th European Solid-State Circuits Conference. ESSCIRC '98, 1998, pp. 308-11.
[86] S. Kavadias, B. Dierickx, D. Scheffer, A. Alaerts, D. Uwaerts, J. Bogaerts, A logarithmic response cmos image sensor with onchip calibration, IEEE Journal of Solid-State Circuits 35 (2000) 1146-1152.

[87] S.-F. Chen, Y.-J. Juang, S.-Y. Huang, and Y.-C. King, Logarithmic cmos image sensor through multi-resolution analog-to-digital conversion, in International Symposium on VLSI Technology, Systems, and Applications, 2003, pp. 227-230.

[88] J. Doge, G. Schonfelder, G.T. Streil, A. Konig, An hdr cmos image sensor with spiking pixels, pixel-level adc, and linear characteristics, Germany IEEE Transactions on Circuits and Systems II: Analog and Digital Signal Processing 49 (2002) 155-158.

[89] N. Tu, R. Hornsey, and S.G. Ingram, Cmos active pixel image sensor with combined linear and logarithmic mode operation, in IEEE Canadian Conference on Electrical and Computer Engineering, vol. 2, May 1998, pp. 754-757.

[90] W. Yang and A. Chiang, A full fill-factor ccd imager with integrated signal processors, in IEEE International Solid-State Circuits Conference, 37th ISSCC., 1990, pp. 218-219.

[91] R. Reich, D. O’Mara, D. Young, A. Loomis, D. Rathman, D. Craig, S. Watson, M. Ulibarri, and B. Kosicki, High-fill-factor, burst-framerate charge-coulped device, in International Electron Devices Meeting, 2001. IEDM Technical Digest, Dec. 2001, pp. 24.6.124.6.4.

[92] S. Chamberlain, Photosensitivity and scanning of silicon image detector arrays, IEEE Journal of Solid-State Circuits 4 (1969) 333-342.

[93] Y.-T. Fan, C.-S. Peng, and C.-Y. Chu, Advanced microlens and color filter process technology for the high-efficiency cmos and ccd image sensors, in Proceedings of the SPIE - The International Society for Optical Engineering, vol. 4115, 2000, pp. 263-74.

[94] A. Bermak, A. Bouzerdoum, and K. Eshraghian, A high fill-factor native logarithmic pixel: simulation, design and layout optimization. in The 2000 IEEE International Symposium on Circuits and Systems. ISCAS 2000 Geneva, vol. 5, 2000, pp. 293-296.

[95] P. Hasler, A. Bandyopadhyay, and P. Smith, A matrix transform imager allowing high-fill factor, in IEEE International Symposium on Circuits and Systems. ISCAS 2002., vol. 3, 2002, pp. 337-340.

[96] H.-C. Chang and Y.-C. King, Tunable injection current compensation architecture for high fill-factor self-buffered active pixel sensor, in IEEE Asia-Pacific Conference on ASIC, 2002., 2002, pp. 101-104.

[97] B. Dierickx, G. Meynants, and D. Scheffer, Near $100 \%$ fill factor cmos active pixels, in IEEE CCD and AIS workshop, 1997.

[98] G. Meynants, B. Dierickx, and D. Scheffer, Cmos active pixel image sensor with ccd performance," in Proceedings of the SPIE - The International Society for Optical Engineering, vol. 3410, 1998, pp. 68-76.

[99] M. Furumiya, H. Ohkubo, Y. Muramatsu, S. Kurosawa, F. Okamoto, Y. Fujimoto, Y. Nakashiba, High-sensitivity and no-crosstalk pixel technology for embedded cmos image sensor, IEEE Transactions on Electron Devices 48 (2001) 2221-2227.

[100] K. Yoon, C. Kim, B. Lee, D. Lee, Single-chip cmos image sensor for mobile applications, IEEE Journal of Solid-State Circuits 37 (2002) 1839-1845.

[101] K.-B. Cho, A. Krymski, and E. Fossum, A 1.2 v micropower cmos active pixel image sensor for portable applications, in IEEE International Solid-State Circuits Conference, ISSCC., 2000, pp. 114-115.

[102] E. Fossum, Low power camera-on-a-chip. using cmos active pixel sensor technology, in IEEE Symposium on Low Power Electronics, 1995, pp. 74-77.

[103] B. Pain, G. Yang, B. Olson, T. Shaw, M. Ortiz, J. Heynssens, C. Wrigley, and C. Ho, A low-power digital camera-on-a-chip implemented in cmos active pixel approach, in Twelfth International Conference On VLSI Design, Jan. 1999, pp. 26-31. 
[104] H.P. Wong, R.T. Chang, E. Crabbe, P.D. Agnello, Cmos active pixel image sensors fabricated using a $1.8-\mathrm{v}, 0.25 \mathrm{~m} \mathrm{cmos}$ technology, IEEE Transactions on Electron Devices 45 (1998) 889-894.

[105] B. Mansoorian, H.-Y. Yee, S. Huang, and E. Fossum, A 250mw, 60 frames/s $1280 \times 720$ pixel $9 \mathrm{~b}$ cmos digital image sensor, in IEEE International Solid-State Circuits Conference. ISSCC, 1999, pp. 312-13.

[106] K.-B. Cho, A. Krymski, and E.R. Fossum, A 1.2 v micropower cmos active pixel image sensor for portable applications, in 2000 IEEE International Solid-State Circuits Conference, 2000, pp. 114-15.

[107] C. Xu, W. Zhang, M. Chan, A low voltage hybrid bulk/soi cmos active pixel image sensor, IEEE Electron Device Letters 22 (2001) 248-250.

[108] J. Ohta, H. Sakata, T. Tokuda, and M. Nunoshita, Low-voltage operation of a cmos image sensor based on pulse frequency modulation, in Proceedings of the SPIE - The International Society for Optical Engineering, vol. 4306, 2001, pp. 319-26.

[109] K.-B. Cho, A.I. Krymski, E.R. Fossum, A 1.5-v 550(w $176 \times 144$ autonomous cmos active pixel image sensor, IEEE Transactions on Electron Devices 50 (2003) 96-105.

[110] E.R.F. Kwang-Bo Cho, Alexander I. Krymski, A 3-pin 1.5-v 550 w $176 \times 144$ selfclocked cmos active pixel image sensor, in IEEE International Symposium on Low Power Electronics and Design, 2001, pp. 316-321.

[111] S. Kawahito, D. Handoko, and Y. Tadokoro, A cmos image sensor with motion vector estimator for low-power image compression, in Proceedings of the 16th IEEE Instrumentation and Measurement Technology Conference. IMTC/99, vol. 1, 1999, pp. 65-70.

[112] B.W.C. Huat, A $128 \times 128$ pixel standard cmos image sensor with electronic shutter, IEEE journal of solid-state circuits 31 (12) (1996) 180-181.

[113] G. Yang, O. Yadid-Pecht, C. Wrigley, and B. Pain, A snap-shot cmos active pixel imager for low-noise, high-speed imaging, in International Electron Devices Meeting. IEDM '98, Dec. 1998, pp. $45-48$.

[114] N. Stevanovic, M. Hillebrand, B. Hosticka, U. Iurgel, and A. Teuner, A high speed camera system based on an image sensor in standard cmos technology, in Proceedings of the 1999 IEEE International Symposium on Circuits and Systems VLSI. ISCAS'99., vol. 5, 1999, pp. $148-51$.

[115] G. Allan, D. Dattani, D. Dykaar, E. Fox, S. Ingram, S. Kaniasz, M. Kiik, B. Li, A. Pavlov, and Q. Tang, High-speed vga cmos image sensor, in Proceedings of the SPIE - The International Society for Optical Engineering, vol. 4306, 2001, pp. 111-18.

[116] N. Stevanovic, M. Hillebrand, B.J. Hosticka, and A. Teuner, A cmos image sensor for high-speed imaging, in IEEE International SolidState Circuits Conference, 2000, pp. 104-5, 449.

[117] N. Stevanovic, M. Hillebrand, B.J. Hosticka, U. Iurgel, and A. Teuner, A high frame rate image sensor in standard cmostechnology, in Proceedings of the 24th European Solid-State Circuits Conference. ESSCIRC' 98., 1998, pp. 316-19.

[118] S. Yoshimura, T. Sugiyama, K. Yonemoto, and K. Ueda, A 48 $\mathrm{kframe} / \mathrm{s}$ cmos image sensor for real-time 3-d sensing and motion detection, in IEEE International Solid-State Circuits conference. ISSCC, 2001, pp. 94-5, 436.

[119] A. Krymski, N.E. Bock, N. Tu, D. Blerkom, and E. Fossum, A highspeed, 240-frame/s, 4.1-mpixel cmos sensor, IEEE Transactions on electron devices, vol. 50, 2003.

[120] J.S. Conde, M. Hillebrand, A. Teuner, N. Stevanovic, U. Iurgel, and B. Hosticka, A smart airbag solution based on a high speed cmos camera system, in International Conference on Image Processing. ICIP 99., vol. 3, 1999, pp. $930-934$.

[121] Y. Oike, M. Ikeda, K. Asada, A cmos image sensor for high-speed active range finding using column-parallel time-domaain adc and position encoder, IEEE Transactions on Electron Devices 50 (2003) $152-158$.
[122] R. Guidash, T.-H. Lee, P. Lee, D. Sackett, C. Drowley, M. Swenson, L. Arbaugh, R. Arbaugh, F. Shapiro, and S. Domer, A $0.6 \mathrm{~m} \mathrm{cmos}$ pinned photodiode color imager technology, in International Electron Devices Meeting, IEDM, Dec. 1997, pp. 927-929.

[123] R. Nixon, S. Kemeny, C. Staller, and E. Fossum, $128 \times 128 \mathrm{cmos}$ photodiode-type active pixel sensor with on-chip timing, control and signal chain electronics, in Charge-Coupled Devices and SolidsState Optical Sensors V, Proc. SPIE, vol. 2415, 1995, pp. 117-123.

[124] I. Takayanagi, J. Nakamura, E.-S. Eid, E. Fossum, K. Nagashima, T. Kunihiro, and H. Yurimoto, A low dark current stacked cmos-aps for charged particle imaging, in International Electron Devices Meeting, IEDM, Dec. 2001, pp. 24.2.1-24.2.4.

[125] I. Takayanagi, J. Nakamura, E. Fossum, K. Nagashima, T. Kunihiro, H. Yurimoto, Dark current reduction in stacked-type cmos-aps for charged particle imaging, IEEE transactions on electron devices 50 (2003) 70-76.

[126] P. Lee and C. Anagnopoulis, Mems/cmos integration and image sensors, in Eleventh Annual IEEE International ASIC Conference, 1998, pp. 381-381.

[127] M. Tabet and R. Hornsey, Cmos image sensor camera with focal plane edge detection, in Canadian Conference on Electrical and Computer Engineering 2001, vol. 2, 2001, pp. 1129-33.

[128] A. Teuner, M. Hillebrand, B. Hosticka, S.-B. Park, J.S. Conde, and N. Stevanovic, Surveillance sensor systems using cmos imagers, in International Conference on Image Analysis and Processing, 1999. Proceedings., 1999, pp. 1124-1127.

[129] Y. Ni, A $256 \times 256$-pixel smart cmos image sensor for line based stereo vision applications, IEEE Journal of Solid-State Circuits 35 (2000) 1055-2000.

[130] S. Habinc. (2001) Active pixel sensors for space applications. [Online]. Available: http://esapub.serin.esa.it/pff/pffv11n1/Habinc. pdf.

[131] J. Goy, B. Courtois, J.M. Karam, and F. Pressecq, Design of an aps cmos image sensor for space applications using standard cad tools and cmos technology, in Proceedings of the SPIE - The International Society for Optical Engineering, vol. 4019, 2000, pp. 145-52.

[132] C. Liebe, E. Dennison, B. Hancock, R. Stirbl, and B. Pain, Active pixel sensor (aps) based star tracker, in IEEE Aerospace Conference., vol. 1, 1998, pp. 119-127.

[133] P. Holl, P. Fischer, P. Klein, G. Lutz, W. Neeser, L. Struder, and N. Wermes, Active pixel matrix for $\mathrm{x}$-ray satellite missions, in Nuclear Science Symposium, 1999. Conference Record., vol. 1, 1999, pp. 171-175.

[134] -, Active pixel matrix for x-ray satellite missions, IEEE Transactions on Nuclear Science, vol. 47, pp. 1421-1425, 2000.

[135] R. Stirbl, B. Pain, T. Cunningham, B.H.J. Heynssens, and C. Wrigley, Advances in ultra-low power, highly integrated, active pixel sensor cmos imagers for space and radiation environments, in Proceedings of the SPIE - The International Society for Optical Engineering, vol. 4547, 2002, pp. 1-10.

[136] E. El-Sayed, Design of radiation hard cmos aps image sensors for space applications, in Proceedings of the Seventeenth National Radio Science Conference. 17th NRSC'2000, 2000, pp. D5/1-9.

[137] B. Hosticka, W. Brockherde, A. Bussmann, T. Heimann, R. Jeremias, A. Kemna, C. Nitta, O. Schrey, Cmos imaging for automotive applications, IEEE Transactions on Electron Devices 50 (2003) 173-183.

[138] B. Wandell, A. Gamal, and B. Girod, Common principles of image acquisition systems and biological vision, in Proceedings of the IEEE, vol. 90(1), 2002, pp. 5-17.

[139] M. Schwarz, R. Hauschild, B. Hosticka, J. Huppertz, T. Kneip, S. Kolnsberg, L. Ewe, H.K. Trieu, Single-chip cmos image sensors for a retina implant system, IEEE Transactions on Circuits and Systems II:Analog and Digital Signal Processing 46 (7) (1999) 870-877. 
[140] H.K. Kim, G. Cho, Y.H. Shin, H.S. Cho, Development and evaluation of a digital radiographic system based on cmos image sensor, IEEE Transactions on Nuclear Science 48 (2001) 662-666.

[141] S.W. Lee, H.K. Kim, Y.H. Shin, A 3-d x-ray microtomographic system with a cmos image sensor, IEEE Transactions on Nuclear Science 48 (2001) 1503-1505.

[142] P. Lechner, R. Hartmann, P. Holl, T. Johannes, P. Klein, J. Kollmer, G. Lutz, R. Richter, L. Struder, P. Fischer, M. Trimpl, J. Ulrici, N. Wermes, A. Castoldi, E. Gatti, and P. Rehak, Active pixel sensor for x-ray imaging spectroscopy, in Nuclear Science Symposium Conference Record, IEEE, vol. 1, 2001, pp. 15-19.

[143] R. Nixon, S.E. Kemeny, and E. Fossum, $256 \times 256 \mathrm{cmos}$ active pixel sensor cameraon-chip, in IEEE International Solid-State Circuits Conference, ISSCC96, Feb. 1996, pp. 178-9, 440.

[144] M. Segawa, M. Ono, S. Musha, Y. Kishimoto, A. Ohashi, A cmos image sensor module applied for a digital still camera utilizing the tab on glass (tog) bonding method, IEEE Transactions on Advanced Packaging 22 (1999) 160-165.

[145] D. Bursky, Cmos megapixel image sensors deliver nearly noise-free pictures, Electronic Design. Penton Publishing, vol. 47, no. 24, pp. 66-68, 1999.

[146] H. Ihara, H. Yamashita, I. Inoue, T. Yamaguchi, N. Nakamura, and H. Nozaki, A $3.7 \times 3.7 \mathrm{~m} /$ sup $2 /$ square pixel cmos image sensor for digital still camera application, in 1IEEE International Solid-State Circuits Conference. ISSCC, 1999, pp. 182-3.

[147] J. Forest and J. Salvi, A review of laser scanning three-dimensional digitisers, in IEEE/RSJ International Conference on Intelligent Robots and System, vol. 1, 2002, pp. $73-78$.

[148] P. Mengel, G. Doemens, and L. Listl, ;Fast range imaging by cmos sensor array through multiple double short time integration (mdsi), in International Conference on Image Processing, 2001., vol. 2, 2001, pp. 169-172.

[149] B. Curless, From range scans to $3 \mathrm{~d}$ models, Computer Graphics 33 (4) (1999).

[150] M. Johannesson and H. Thorngren. (2001) Advances in cmos technology enables higher speed true 3d-measurements. [Online]. Available: https://www.machinevisiononline.org/public/articles/ ivp1.pdf.

[151] Y. Oike, M. Ikeda and K. Asada, High-speed position detector using new row-parallel architecture for fast collision prevention system, Proceedings of the 2003 International Symposium on Circuits and Systems, 2003. ISCAS 03., vol. 4, pp. $788-791,2003$.

[152] W. Brockherde, B.J. Hosticka, M. Petermann, M. Schanz, and R. Spors, Smart 2048-pixel linear cmos image sensor, in Proceedings of the 24th EuropeanSolid-State Circuits Conference. ESSCIRC '98, 1998, pp. 212-15. 